\title{
Exact trajectory solutions of a spherical microswimmer under flow and external fields
}

\author{
M. Guedda, ${ }^{1}$ J. Chaiboub, ${ }^{1}$ M. Benlahsen, ${ }^{2}$ and C. Misbah ${ }^{3}$ \\ ${ }^{1}$ LAMFA, CNRS UMR 7352, Univ. Picardie Jules Verne, F-80039 Amiens, France \\ ${ }^{2}$ LPMC, Univ. Picardie Jules Verne, F-80039 Amiens, France \\ ${ }^{3}$ Univ. Grenoble Alpes, CNRS, LIPhy, F-38000 Grenoble, France
}

The main objective of the present study is the derivation of exact analytical expressions for the orientation and trajectory of a spherical microswimmer submitted to general linear flows and to an external (gravitational or magnetic) force field, a problem known as gyrotaxis or magnetotaxis. We consider linear shear, hyperbolic, solid-rotation and stagnation flows. The evolution equations of the swimmer orientation and its position are nonlinear and analytical results are the exception rather than the rule. Most of available results for cell orientation and trajectories are obtained numerically. The solution for the swimmer orientation is inspired from a method due to Bretherton, initially developed for a different nonlinear equation. We show here that this method can be generalized to our evolution equation. We will see that the swimmer under flow exhibits both run (a motion where the orientation angle is kept constant with time) and tumble (the orientation angle is cyclic with time) regimes, and a variety of cell trajectories are extracted analytically, such as parabolic, elliptic, helical, and so on. This study offers a framework to generalize the results to other types of flows.

PACS Numbers: 


\section{INTRODUCTION}

Swimmers range from self-propelled microorganisms to macroscopic animals such fishs. Macroscopic organisms use inertia in their motion, whereas microorganisms, like bacteria, spermatozoa, micro-algae etc, have a low enough Reynolds number (Re) for inertial effects to be ignored in describing their locomotion.

In the zero Re limit, the governing equations of fluid motion are linear (the Stokes equations) and invariant under time reversal. As a result, microorganisms must deploy non reciprocal deformations in time in order to move forward (the scallop theorem or the reciprocal theorem) [1], [2]. Microorganisms, like spermatozoa, bacteria and microalgae, can move with the help of flagella or cilia [3], [4] thanks to non reciprocal elementary strokes. Other cells may use ample shape deformations, known as amoeboid [5], for their locomotion.

Many microorganisms respond to both flow and other stimuli (e.g. external force), such as gravity (gyrotaxis), magnetic field (magnetotaxis), light (phototaxis), and so on. The interplay between flow and external force leads to a variety of complex motions. Most of trajectories of these microswimmers have been so far obtained numerically, as described below.

Under flow and an external field the orientation vector (denoted as $\boldsymbol{p}$ ) and the position (denoted as $\mathbf{x}$ ) of a microswimmer with a spherical shape assumption are described by

$$
\dot{\boldsymbol{p}}=\frac{1}{2} \boldsymbol{\omega} \times \boldsymbol{p}+\lambda\left[\dot{\boldsymbol{j}}^{\star}-\left(\boldsymbol{p} \cdot \dot{j}^{\star}\right) \boldsymbol{p}\right], \quad \dot{\mathbf{x}}=\mathbf{u}_{0}(\mathbf{x})+\mathbf{v}_{\mathbf{s}},
$$

where $\boldsymbol{\omega}$ is the fluid vorticity vector, $\boldsymbol{j}^{\star}$ accounts for the applied external field characterized by $\lambda(\lambda=0$ in the absence of the external field), proportional, for example, to gravity for a heavy bottom particle (i.e. when the mass center differs from the sphere center [6-8]), $\mathbf{v}_{\mathbf{s}}=v_{s} \boldsymbol{p}$ is the swimming velocity and $\mathbf{u}_{\mathbf{0}}$ is the applied flow. Brenner [6, 7] investigated the effect of shear flow for the cases where $\boldsymbol{\omega}$ and $\boldsymbol{j}^{\star}$ are either perpendicular or parallel, whereas Hall and Busenberg[8] studied the general case where the angle between $\boldsymbol{\omega}$ and $\boldsymbol{j}^{\star}$ can be arbitrary. Their results essentially show that the particle either tends to a fixed orientation or describes a periodic motion. These two motions will be referred to as run and tumble motions. This is not to be confused with run-and-tumble trajectory where the straight trajectory is interrupted by sudden changes of orientation. Here we mean by 'run' that the particle keeps always a given orientation, and by 'tumbling' that the particle undergoes a cyclic motion. Brenner [6, 7] determined the equilibrium orientation (corresponding to $d \boldsymbol{p} / d t=0$ ), and derived the condition under which an equilibrium orientation exists and is stable. When the steady orientation ceases to exist the particle is expected to tumble. Hall and Busenberg [8] used an application of the Poincaré-Bendixon stability theorem [9] to predict that the particle achieves a unique stable terminal orientation when the applied field and vorticity are not perpendicular. In the same spirit as in [6-8], Pedley and Kessler [10] have shown how to calculate equilibrium orientations of spheroidal microorganisms in a general flow and how to predict run and tumble motions. The corresponding evolution equation for spheroidal shapes is different from (1) and contains an additional cubic term for $\boldsymbol{p}$ due to the straining part of the flow. They provided a general expression for equilibrium orientations in terms of polar angles and discussed their stability. However, in all these studies [6-8, 10] no analytical solution was provided neither for the orientation angle in the tumbling regime nor for the particle trajectory.

It has been observed that the interplay between fluid vorticity and external field can lead to several consequences regarding swimmer distribution under non linear flows, such as a Poiseuille flow. More precisely, Kessler [11] has studied experimentally and theoretically the trajectory and spatial distribution of Chlamydomonas or Dunaliella cells, which are subject to a torque induced by gravity (Gyrotaxis) in a Poiseuille flow. It is shown that when shear and gravity torques balance, cells keep a fixed orientation (run regime) for sufficiently small vorticity and accumulate near the center of the vertical tube with a downward flow. For upwards flow, the particles move away from the axis and accumulate at the periphery of the cylinder, where they perform tumbling motion (if the vorticity is large enough). Kessler [11] has provided the run solution (constant orientation) and the condition for its stability. No analytical solution to the orientation vector or to the particle trajectory is given. Kessler provided, however, a nice solution for the concentration field supporting his observation (accumulation at the center or at the periphery).

In [12], Zottl and Stark have considered a spherical swimmer in Poiseuille flow in the absence of external force $(\lambda=0$ in Eq.(1)), besides noise. The authors showed that the deterministic part of the trajectory evolution equations can be mapped onto a Hamiltonian system and different interesting behaviors of swimmer trajectories are determined. In Ref.[13], the authors presented an experimental study of bioconvection and cell dispersion in a horizontal tubes with and without flow. Numerical trajectories are presented for a spherical cell (in Poiseuille and plug flows), by making use of equations of type (1) in which the cell is assumed to swim with a fixed orientation (run regime). However, no analytical solution of trajectory is presented. In [14], the distribution of gyrotactic prolate ellipsoidal microorganisms in a steady vortical flow is examined. Numerical simulations showed a rich variety of trajectories, including closed and spiral trajectories, that are highly dependent upon two physical parameters: the swimming speed relative to a characteristic fluid speed and the magnitude of the gyrotactic torque. Very recently, the effect of motility and shape on the microswimmer dynamics in a two dimensional axisymmetric (stationary Lamb-Oseen) vortex flow is studied [15]. The swimmer is modeled as an ellipsoidal particle having a given swimming direction. By using a dynamical system theory (Poincaré-Bendixson theorem), rich dynamics is obtained by classifying the fixed-point structures as a function of particle shape and the relative swimming velocity. Different trajectories are obtained numerically showing bounded and unbounded trajectories and transitions among them. In particular, it is found that prolate microswimmers tend to 
align parallel to the velocity field, while oblate microswimmers tend to remain perpendicular to it. For spherical swimmers, a homogeneous distribution is obtained. Matsunaga et al. [16], presented a method to control particle trajectories in a Poiseuille flow thanks to a static magnetic field. The authors showed, by numerical simulations, that the ellipsoidal magnetic particle can be focused to an arbitrary transverse position. Equation (1) in the presence of gravity and fluid acceleration (solid body rotation) parallel to gravity has been studied regarding migration of plankton [17], [18]. An approximate solution for the particle trajectory (see Section V for more detail) is provided, allowing for an exact solution determination.

Orientation and trajectory of spherical micro-organisms in one dimensional vertical shear flow and under gravitactic and intrinsic torques are studied numerically in Ref. [19]. In particular, the effect of the intrinsic torque on the particle orientation and trajectory are investigated. Analytical solutions for trajectories are obtained only if the cell has a fixed orientation (run regime). Thorn et al. [20] have considered the motion and the transport of spherical particles in different types of flows in $x-y$ plane (shear flow, solid-body rotation, straining flow), and for arbitrary angle between the fluid vorticity and the external field (due to gravity), with and without additive stochastic reorientation. In the deterministic case, expressions of the equilibrium orientations of (1) are presented and their stability is discussed. Particle trajectories are obtained numerically in the tumbling regime.

In summary, in all the above studies, except for run motion, or in the absence of external force, the determination of the orientation vector as a function of time and particle trajectories have been obtained numerically. Here, we will examine several cases with linear flows (shear and straining flows, rigid rotation...) and external fields with different orientations. We will show that in almost all cases studied here explicit analytical solutions can be obtained both for particle orientation and trajectories.

The determination of exact solutions for the orientation was motivated by our previous study in a different context (vesicle and red blood cells under flow), where we had shown that the problem could be mapped onto that of a heavy bottom spherical particle (in higher dimensions) [21]. Since we had earlier derived an exact analytical solution for vesicles [22], this allowed us to show exact solutions (not known before) describing rigid heavy-bottom particles orientation in the presence of linear shear flow. These solutions can be now generalized to different flows and orientations of external fields. The first step here will be to calculate the particle orientation, by presenting a general method à la Bretherton, instead of using the strategy followed for our analytical solution [22]. This new way allows for an easier generalization to different types of equations and external fields (flow and external force). Two main types of motion will be characterized: the run-like solution and the tumble-like solution. Once, the orientations vector solution is found, our second step consists in extracting analytically the particle trajectories. A rich variety of trajectory patterns will be revealed.

\section{ORIENTATION EVOLUTION EQUATION}

We focus on the dynamics of an isolated rigid spherical (magnetic or gyrotactic) swimmer in general linear flows and in the presence of an external field. The swimmer moves with a velocity $\mathbf{v}_{s}=v_{s} \boldsymbol{p}$, where $\boldsymbol{p}$ is the unit particle vector orientation and $v_{s}$, the magnitude of the swimming velocity, is assumed to be constant. It is assumed here that the orientation of the speed is at any instant oriented along $\boldsymbol{p}$. In the absence of rotary Brownian motion, the evolution of the unit orientation vector (or swimming direction) $\boldsymbol{p}$ and position $\mathbf{x}=(x, y, z)$ of the particle can be modeled (at low Reynolds number) by the equations [6, 8, 23]

$$
\begin{gathered}
\dot{\boldsymbol{p}}=\frac{1}{2} \boldsymbol{\omega} \times \boldsymbol{p}+\frac{\mathscr{M}}{8 \pi \mu r^{3}}[\boldsymbol{B}-(\boldsymbol{p} . \boldsymbol{B}) \boldsymbol{p}], \\
\dot{\mathbf{x}}=\mathbf{u}_{0}(\mathbf{x})+v_{s} \boldsymbol{p},
\end{gathered}
$$

where $\mathbf{u}_{0}$ is the fluid velocity and $\boldsymbol{\omega}=\nabla \times \mathbf{u}_{0}$ is the fluid vorticity vector. We use Cartesian coordinates $(x, y, z)$, with corresponding unit vectors denoted by $(\boldsymbol{i}, \boldsymbol{j}, \boldsymbol{k})$. Note that $\boldsymbol{\omega} \times \boldsymbol{p}$ can be replaced by $\boldsymbol{\Omega} . \boldsymbol{p}$, where matrix $\boldsymbol{\Omega}$ is the vorticity tensor given by

$$
\boldsymbol{\Omega}=\left(\begin{array}{ccc}
0 & -\omega_{3} & \omega_{2} \\
\omega_{3} & 0 & -\omega_{1} \\
-\omega_{2} & \omega_{1} & 0
\end{array}\right)
$$

where $\boldsymbol{\omega}=\left(\omega_{1}, \omega_{2}, \omega_{3}\right)$. The other parameters appearing in the above equations are the radius of the spherical particle, $r$, the kinematic viscosity of the suspending fluid, $\mu, \boldsymbol{B}$ is the uniform external field vector and $\mathscr{M}=|\boldsymbol{M}|$, where $\boldsymbol{M}$ is the dipole moment of the particle.

We introduce the unit vector $\boldsymbol{j}^{\star}$ such that $\boldsymbol{B}=\boldsymbol{B} \boldsymbol{j}^{\star}$. Therefore, Eq. (2) can be rewritten as

$$
\dot{\boldsymbol{p}}=\frac{1}{2}\left[\boldsymbol{\Omega} \cdot \boldsymbol{p}+\lambda\left(\boldsymbol{j}^{\star}-\boldsymbol{j}^{\star} \cdot \boldsymbol{p} \boldsymbol{p}\right)\right],
$$


where $\lambda$ is a real physical parameter given by

$$
\lambda=\frac{\mathscr{M} B}{4 \pi \mu r^{3}}
$$

for a magnetic swimmer. The same equation is derived for a spherical gyrotactic particle (called heavy-bottom particle [6, 7, 24]), with

$$
\lambda=\frac{\rho h g}{3 \mu},
$$

where $\rho$ is the cell density, $h$ is the center of mass offset from the geometrical center and $g$ is the acceleration due to gravity. Quantity $\lambda^{-1}$ represents the typical characteristic time a perturbed particle takes to return to orientation $\boldsymbol{j}^{\star}$ if $\boldsymbol{\Omega}=0$ (i. e., $\boldsymbol{\omega}=0$ ).

As mentioned in the introduction, Eq. (5), and more general equations for spheroidal particles, have been studied by Brenner $[6,7]$ and Hall and Busenberg [8]. Two types of motions are obtained depending on parameter $\chi=\lambda / \omega$, where $\omega$ is the strength of $\boldsymbol{\omega}$, and $\psi$ the angle between $\boldsymbol{\omega}$ and $\boldsymbol{j}^{\star}$. If $\psi \neq \pi / 2$, or if $\psi=\pi / 2$ and $\chi>1$, orientation $\boldsymbol{p}$ tends in time to a certain fixed orientation irrespective of its initial orientation (run regime). For $\psi=\pi / 2$ and $\chi<1$, orientation $\boldsymbol{p}$ describes one of an infinite family of periodic closed orbits (tumble regime). In the tumbling regime no analytical solution was given. The extraction of analytical solutions for the orientation vector (both for run and tumble regimes) is the first objective of the present paper.

In what follows we shall see how to exhibit exact solutions to Eq. (5), by using an idea due to Bretherton [25]. The next Section introduces a simple and general method to analytically solve a class of equations of the type (5), for arbitrary parameter $\lambda$ and vorticity tensor $\boldsymbol{\Omega}$. Another important new feature that constitutes the second objective of the paper, is to derive analytically the particle trajectory in linear flows, by exploiting the exact analytical expression of the particle orientation.

\section{A UNIFIED METHOD FOR THE ORIENTATION EQUATION}

Recently, as mentioned before, we have studied the dynamics of quasi spherical vesicles under shear flow [21], and have shown that the shape evolution equation can be mapped onto that of a rigid sphere similar to Eq. (5), with appropriate $\lambda$, in five dimensions and with an appropriate fictitious vorticity $\boldsymbol{\Omega}$. Based on this mapping and on our previous exact analytical solutions for vesicle [22], we can solve Eq. (5). Here, instead of the method of [21], we provide a more general analytical approach which can be more easily extended to other physical situations (such as arbitrary orientations of the external field).

\section{A. Bretherton approach}

After the famous Jeffery work [26] on dynamics of a rigid ellipsoid under shear flow, Bretherton [25] extended it to particles with a more general shape. More precisely, he showed that any rigid non spherical particle in Stokes flow obeys the following evolution equation

$$
\dot{\boldsymbol{p}}=\mathscr{S} \cdot \boldsymbol{p}+\beta_{\mathrm{s}}[\mathbf{E} \cdot \boldsymbol{p}-(\boldsymbol{p} . \mathbf{E} \cdot \boldsymbol{p}) \boldsymbol{p}],
$$

or, equivalently,

$$
\dot{\boldsymbol{p}}=\mathbb{A} \cdot \boldsymbol{p}-(\boldsymbol{p} \cdot \mathbb{A} \cdot \boldsymbol{p}) \boldsymbol{p},
$$

with $\mathbb{A}=\mathscr{S}+\beta_{s} \mathbf{E}, \mathscr{S}=\left(\nabla \mathbf{u}_{0}-\nabla \mathbf{u}_{0}^{T}\right) / 2$ and $\mathbf{E}=\left(\nabla \mathbf{u}_{0}+\nabla \mathbf{u}_{0}^{T}\right) / 2$ are the vorticity and rate-of-strain tensors, respectively, and parameter $\beta_{\mathrm{s}}$ is the shape factor (its value is zero for a sphere and different from zero for any other shape). In Eq. (8), $\boldsymbol{p}$ is the orientation vector taken to be along the major semi-axis.

To solve (9), Bretherton [25] insightfully noticed that the term (p.A.p) $\boldsymbol{p}$ in Eq. (9), which is parallel to $\boldsymbol{p}$, acts only to conserve the unit length of $\boldsymbol{p}$ and does not affect its orientation. He showed that the temporal evolution of the orientation vector $\boldsymbol{p}$ can be deduced from any vector $\boldsymbol{q}$ that is parallel to $\boldsymbol{p}$ and evolves according to

$$
\dot{\boldsymbol{q}}=\mathbb{A} \cdot \boldsymbol{q}
$$

and then the solution to Eq. (9) follows

$$
\boldsymbol{p}=\frac{\boldsymbol{q}}{|\boldsymbol{q}|}
$$


where

$$
\boldsymbol{q}=\exp (\mathbb{A} t) \boldsymbol{q}(0)
$$

where exp denotes the matrix exponential.

In summary, from a mathematical point of view, the main idea of Bretherton approach is to use the Ansatz

$$
\boldsymbol{p}=\alpha(t) \boldsymbol{q}
$$

that reduces Eq. (9) to the ordinary differential equation satisfied by $\alpha$ :

$$
\frac{d \alpha}{d t}=\alpha^{3}(\boldsymbol{q} \cdot \mathbb{A} \cdot \boldsymbol{q}),
$$

which is easy to integrate. The Bretherton approach is extended and adapted to solve our problem in a closed form.

\section{B. Extension to the present problem}

It is not obvious to adopt a priori the method of previous Section to our equation (Eq. (5)). Appendix A describes how to handle this question. As explained in this appendix for the particular and interesting case in which $\boldsymbol{j}^{\star}$ and $\boldsymbol{p}_{0}$ are perpendicular (this orthogonality will naturally emerge, as discussed below), the general solution of Eq. (5) can be written as

$$
\boldsymbol{p}(t)=\boldsymbol{p}_{0}+\frac{\exp \left(\frac{1}{2} \tilde{\boldsymbol{\Omega}}_{0} t\right) \boldsymbol{q}(0)}{\alpha^{-1}(0)+\frac{\lambda}{2} \int_{0}^{t} \boldsymbol{j}^{\star} \cdot \exp \left(\frac{1}{2} \tilde{\boldsymbol{\Omega}}_{0} s\right) \boldsymbol{q}(0) d s},
$$

with

$$
p_{0}=\xi \omega+\frac{\lambda}{\omega^{2}} \boldsymbol{\omega} \times j^{\star}
$$

where $\xi \in \mathbb{R}$, and where matrix $\tilde{\Omega}_{0}$ is defined by

$$
\tilde{\boldsymbol{\Omega}}_{0}=\boldsymbol{\Omega}-\lambda \boldsymbol{p}_{0} \boldsymbol{j}^{\star \mathrm{T}} .
$$

It can be shown that parameter $\xi$ plays the same role as the Jeffery orbit constant (it can be checked in our explicit calculations below that it can be absorbed into the Jeffery constant). $\alpha(0)$ and $\boldsymbol{q}(0)$ (initial conditions) have to be chosen such that $|\boldsymbol{p}|=1$.

The general solution (15) is obtained by employing the Ansatz

$$
\boldsymbol{p}=\boldsymbol{p}_{0}+\alpha(t) \boldsymbol{q}
$$

\section{DYNAMICS OF A MICROSWIMMER IN 1D UNIDIRECTIONAL FLOWS}

The goal now is to draw a global picture of a microswimmer orientation and its trajectory, for a class of external stimuli and linear flow geometries. We study in this Section flows which have only one nonzero velocity component (unidirectional flows). The bidirectional flow will considered in the next Section.

\section{A. Swimming direction in 1d-linear shear flow}

We will see that the swimmer will exhibit a fixed orientation (run regime) or a cyclic one (tumbling regime) in the course of time, depending on flow strength. The imposed fluid velocity has the representation

$$
\mathbf{u}_{0}=(\dot{\gamma} y, 0,0),
$$

$\dot{\gamma}$ being the steady shear rate. We also assume that the external field is parallel to $\boldsymbol{j}$, so that $\boldsymbol{j}^{\star}=\boldsymbol{j}$. The fluid vorticity is given by $\boldsymbol{\omega}=-\dot{\gamma} \boldsymbol{k}$. From (16) we get the one-parameter family:

$$
\boldsymbol{p}_{0}=\xi \boldsymbol{k}+\tilde{\lambda} \boldsymbol{i}
$$

where $\tilde{\lambda}=\lambda / \dot{\gamma}\left(=-\lambda / \omega_{3}\right)$. The exact expression of the orientation vector is calculated from (15) by taking $\boldsymbol{p}_{0}=\tilde{\lambda} \boldsymbol{i}$ (actually it can be checked that $\xi$ entering in (20) is unimportant and can be set to zero). We assume that $\lambda$ (or $\tilde{\lambda}$ ) is positive. Two cases can be distinguished: 
1. For $\tilde{\lambda}>1$,

$$
p_{x}=\tilde{\lambda}-\frac{\Lambda^{2}}{\tilde{\lambda}} \frac{\cosh \left(\frac{\Lambda \dot{\gamma} t}{2}\right)}{a+\cosh \left(\frac{\Lambda \dot{\gamma} t}{2}\right)}, \quad p_{y}=\frac{\Lambda}{\tilde{\lambda}} \frac{\sinh \left(\frac{\Lambda \dot{\gamma} t}{2}\right)}{a+\cosh \left(\frac{\Lambda \dot{\gamma} t}{2}\right)}, \quad p_{z}=\frac{\Lambda c}{\tilde{\lambda}} \frac{1}{a+\cosh \left(\frac{\Lambda \dot{\gamma} t}{2}\right)},
$$

where

$$
\Lambda=\sqrt{\left|\tilde{\lambda}^{2}-1\right|}
$$

and $a$ and $c$ are integration constants determined by the initial orientation and satisfy (due to normalization condition; note that $\tilde{\lambda}^{2} a^{2} \leq 1$ )

$$
c= \pm \sqrt{1-\tilde{\lambda}^{2} a^{2}}
$$

2. If $\tilde{\lambda}<1$,

$$
\boldsymbol{p}_{x}=\tilde{\lambda}+\frac{\Lambda^{2}}{\tilde{\lambda}} \frac{\sin (\Lambda \dot{\gamma} t / 2)}{a+\sin (\Lambda \dot{\gamma} t / 2)}, \quad \boldsymbol{p}_{y}=\frac{\Lambda}{\tilde{\lambda}} \frac{\cos (\Lambda \dot{\gamma} t / 2)}{a+\sin (\Lambda \dot{\gamma} t / 2)}, \quad \boldsymbol{p}_{z}=\frac{\Lambda c}{\tilde{\lambda}^{2}} \frac{1}{a+\sin (\Lambda \dot{\gamma} t / 2)},
$$

where $\Lambda$ is defined in (22), $a$ is a real parameter and $c=\frac{\Gamma}{|\Gamma|} \sqrt{\Gamma^{2}-\tilde{\lambda}^{2}}$, in which $\Gamma=\tilde{\lambda}^{2} a$ satisfying $|\Gamma| \geq \tilde{\lambda}$.

Now, we are ready to easily analyze the dynamics of $\boldsymbol{p}$.

Case $1: \tilde{\lambda}>1$. In the limit $t \rightarrow \infty$,

$$
\boldsymbol{p} \rightarrow \tilde{\lambda}^{-1} \boldsymbol{i}+\sqrt{1-\tilde{\lambda}^{-2}} \boldsymbol{j}=\boldsymbol{p}_{\mathrm{eq}}
$$

This is a pure run motion, showing that the particle orientation tends to the unique stable terminal orientation (irrespective of its initial orientation). During the run motion, the alignment angle $0<\phi<\pi / 2$, between the microswimmer orientation (or $\boldsymbol{p}$ ) and the external force (or $\boldsymbol{j}$ ), is given at any instant by

$$
\phi_{\lambda}=\arctan \left(\Lambda^{-1} \frac{a \tilde{\lambda}^{2}+\cosh \left(\frac{\Lambda \dot{\gamma} t}{2}\right)}{\sinh \left(\frac{\Lambda \dot{\gamma}}{2}\right)}\right) .
$$

For large $t, \phi_{\lambda}$ approaches

$$
\phi_{\lambda}(\infty)=\arctan \left(\Lambda^{-1}\right)=\sin ^{-1}\left(\tilde{\lambda}^{-1}\right) .
$$

Since $\boldsymbol{p}_{\text {eq }}$ is perpendicular to $\boldsymbol{\omega}$ the swimmer is unable to rotate for large $t$.

Case $2: \tilde{\lambda}<1$. In this situation, the equilibrium orientation angle (27) does not exist and, as it is known and transparent from Eq. (24), that the particle performs tumbling motions or periodic orbits (see Figure 1) with period

$$
T=\frac{4 \pi}{\dot{\gamma} \sqrt{1-\lambda^{2}}}
$$

For the particular case where there is no preferred swimming direction $\lambda=0$ (strong rotation or absence of external torque), we get

$$
p_{x}=a_{0} \cos \left(\dot{\gamma} t / 2+\theta_{0}\right), \quad p_{y}=a_{0} \sin \left(\dot{\gamma} t / 2+\theta_{0}\right), \quad p_{z}= \pm \sqrt{1-a_{0}^{2}}
$$

where $\theta_{0}$ and $a_{0}$ are constant parameters (depending on initial orientation), showing that $\boldsymbol{p}$ describes a family of circular orbits about the vertical $z$-axis [27].

\section{B. Swimming Trajectories in 1d linear shear flow}

The aim of this Section is to examine the long-term transport of the particle in simple shear flow. Recall that the time evolution of its center-of-mass position is given by

$$
\dot{\mathbf{x}}=\mathbf{u}_{0}(\mathbf{x})+\mathbf{v}_{\mathbf{s}},
$$




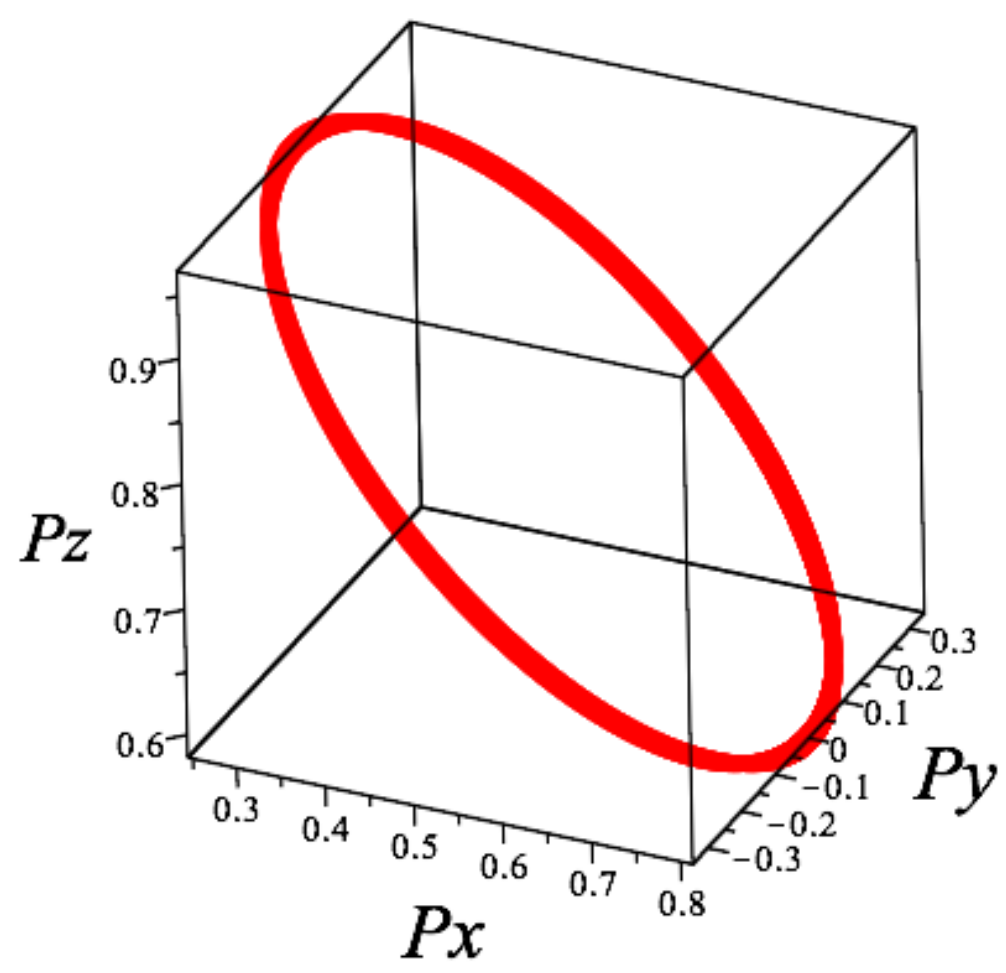

FIG. 1. Motion of orientation $p$ in the tumbling case. $\tilde{\lambda}=0.5, \dot{\gamma}=1$ and $a=4$.

in which vector $\mathbf{v}_{\mathbf{s}}=v_{s} \boldsymbol{p}$ is the swimming velocity vector relative to the fluid and magnitude $v_{s}=\left|\mathbf{v}_{\mathbf{s}}\right|$ is assumed to be constant.

It is well known that several physical forces may influence particle trajectories. For instance, as mentioned in the introduction, a gyrotactic cell may swim at a fixed orientation or it may tumble [10,11]. Taking advantage of the explicit expressions of the orientation vector $\boldsymbol{p}$, we derive here exact expressions for the swimmer position $\boldsymbol{x}=(x, y, z)$, by solving the set of equations

$$
\frac{d x}{d t}=\dot{\gamma} y+v_{s} \boldsymbol{p}_{x}, \quad \frac{d y}{d t}=v_{s} \boldsymbol{p}_{y}, \quad \frac{d z}{d t}=v_{s} \boldsymbol{p}_{z}
$$

\section{Trajectories in the run regime}

We assume that the swimmer is at (stable) equilibrium orientation $\boldsymbol{p}=\boldsymbol{p}_{\text {eq }}$ (see Eq. (25)). The $y$-equation (31) is solved by

$$
y(t)=y(0)+v_{s} \sqrt{1-\tilde{\lambda}^{-2}} t
$$

from which one deduces

$$
x(t)=x(0)+\left(\dot{\gamma} y(0)+v_{s} \tilde{\lambda}^{-1}\right) t+\frac{\dot{\gamma} v_{s}}{2} \sqrt{1-\tilde{\lambda}^{-2}} t^{2} .
$$

The $z$-component is constant; $z(t)=z(0)$. Eqs. (32) and (33) show that coordinates $x$ and $y$ tend to infinity with time $t$ and that the $x-y$ plane projection of the trajectory has the form

$$
x-x(0)=\frac{\dot{\gamma} y(0)+v_{s} \tilde{\lambda}^{-1}}{v_{s} \sqrt{1-\tilde{\lambda}^{-2}}}(y-y(0))+\frac{\dot{\gamma}}{2 v_{s} \sqrt{1-\tilde{\lambda}^{-2}}}(y-y(0))^{2},
$$

which describes a parabola. Note that the long-time behavior of the $x-y$ plane projection trajectory is given by

$$
x \approx \frac{\lambda}{2 v_{s} \Lambda} y^{2} .
$$

This result is identical to that of a spherical gyrotactic particle studied in [20]. 
For general run regime, the trajectories are described by (recall that $a^{2} \tilde{\lambda}^{2} \leq 1$ )

$$
\begin{aligned}
x(t)=x(0) & +\left(\dot{\gamma} y(0)+\frac{v_{s}}{\tilde{\lambda}}-\frac{2 v_{s}}{\tilde{\lambda}} \ln (a+1)\right) t+\frac{2 v_{s}}{\tilde{\lambda}} \int_{0}^{t} \ln \left(a+\cosh \left(\frac{\dot{\gamma} \Lambda}{2} s\right)\right) d s \\
& +\frac{4 a}{\sqrt{1-a^{2}}} \frac{v_{s}\left(\tilde{\lambda}^{2}-1\right)}{\Lambda \lambda}\left\{\tan ^{-1}\left(\frac{1}{\sqrt{1-a^{2}}}\left(a+e^{(\dot{\gamma} \Lambda / 2) t}\right)\right)-\tan ^{-1}\left(\frac{1}{\sqrt{1-a^{2}}}(a+1)\right)\right\}, \\
y(t)=y(0) & +\frac{2 v_{s}}{\lambda} \ln \left(\frac{a+\cosh \left(\frac{\dot{\gamma} \Lambda}{2}\right) t}{a+1}\right) .
\end{aligned}
$$

The $z$-component is given by

$$
z(t)=z(0)+\frac{4 \sqrt{1-\tilde{\lambda}^{2} a^{2}}}{\sqrt{1-a^{2}}} \frac{v_{s}}{\lambda}\left\{\tan ^{-1}\left(\frac{1}{\sqrt{1-a^{2}}}\left(a+e^{(\dot{\gamma} \Lambda / 2) t}\right)\right)-\tan ^{-1}\left(\frac{1}{\sqrt{1-a^{2}}}(a+1)\right)\right\} .
$$

For large values of $t$ the $z$ - component tends to a constant and the components $x$ and $y$ approach a parabola satisfying Eq. (35) (trajectories are parallel to the $x-y$ plane).

\section{Trajectories in the tumbling regime}

Let us first consider two particular examples. The first case corresponds to the situation where the swimming speed is very small in comparison to the imposed flow speed. Neglecting terms proportional to $v_{s}$ in (36), meaning that the particle is simply advected by the flow, we have

$$
x(t)=x(0)+\dot{\gamma} y(0) t, \quad y(t)=y(0), \quad z(t)=z(0),
$$

meaning that the particle migrates perpendicular to the applied external field and parallel to the $x-y$ plane.

In the second example we assume that $\lambda=0$ (no external force). In that case we obtain from (29) and (31)

$$
\begin{gathered}
x-x(0)=\left\{\dot{\gamma} y(0)+2 a_{0} v_{s} \cos \left(\theta_{0}\right)\right\} t+\frac{4 a v_{s}}{\dot{\gamma}}\left(\sin \left(\theta_{0}\right)-\sin \left(\dot{\gamma} t / 2+\theta_{0}\right)\right), \\
y-y(0)=\frac{2 a_{0} v_{s}}{\dot{\gamma}}\left(\cos \left(\theta_{0}\right)-\cos \left(\dot{\gamma} t / 2+\theta_{0}\right)\right),
\end{gathered}
$$

and

$$
z-z(0)= \pm v_{s} \sqrt{1-a_{0}^{2}} t
$$

leading to two different scenarios (recall that $a_{0}$ and $\theta_{0}$ are constant parameters depending on initial orientation). For example if $a_{0}^{2} \neq 1$, we deduce that if $\left|\dot{\gamma} y(0) / 2 a_{0} v_{s}\right| \leq 1$, the particle may follow a periodic trajectory. To be more precise, if $\dot{\gamma} y(0)+2 a_{0} v_{s} \cos \left(\theta_{0}\right)=0$ the swimmer moves along a helical path (with the helix pitch along the $z$-axis, see Figure 2 ), while if $\left|\dot{\gamma} y(0) / 2 a_{0} v_{s}\right|>1$, or $\dot{\gamma} y(0)+2 a_{0} v_{s} \cos \left(\theta_{0}\right) \neq 0$, the swimmer trajectory is unbounded along both $x$ and $z$ directions (Figure 3). However, in the frame moving along $x$ with velocity $\dot{\gamma} y(0)+2 a_{0} v_{s} \cos \left(\theta_{0}\right)$ the trajectory is a helix with the pitch along $z$; the helix wanders around the $z$-axis, as in Figure 2. For the particular case where $\lambda=0$, which corresponds to the absence of external torque, the spherical particle tumbles and its trajectory describes a cycloid in the shear plane.

In [28], ten Hagen et al. have derived similar expressions for $x$ and $y$, Eqs. (39) and (40), to describe two-dimensional trajectories of a self-propelled spherical particle under linear shear flow and rotates in a magnetic field parallel to the fluid vorticity. In their case the applied magnetic torque is constant and only adds up to vorticity in Eq. (5). Their equation is linear in $\boldsymbol{p}(\lambda=0)$ and the total angular velocity for the particle for this case is given by the sum of the fluid vorticity and the external torque; $\boldsymbol{O}=(-\dot{\gamma} / 2+M) \boldsymbol{k}$, where $M$ is the magnitude of the external torque. 


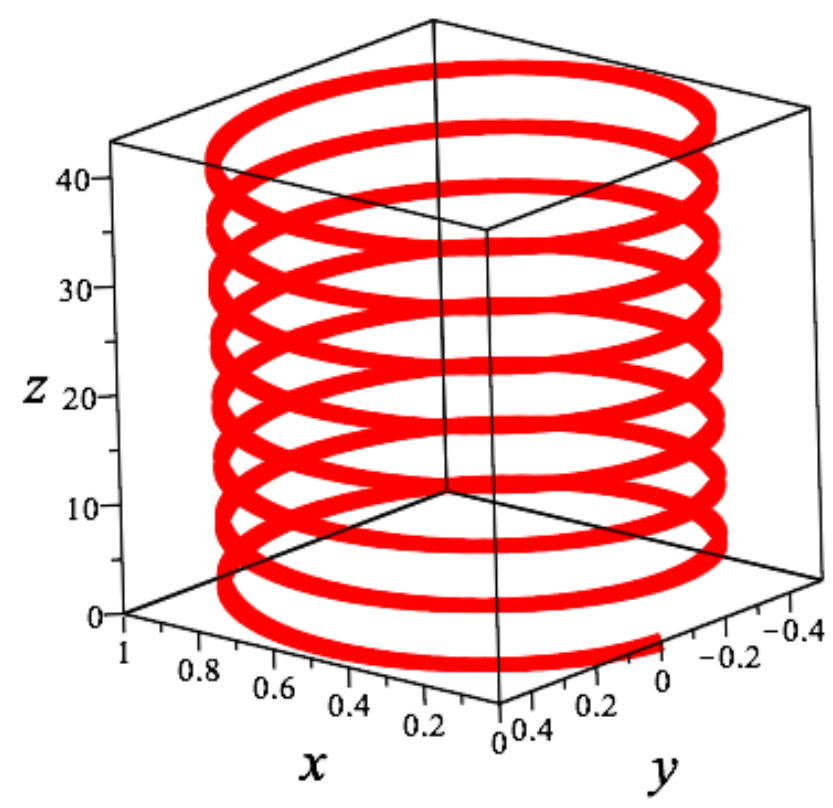

FIG. 2. Example of a periodic trajectory in the absence of external torque (Eqs. (39)-(41)). Parameters are $\theta_{0}=\pi / 2, a_{0}=0.5, v_{s}=1$ and $\dot{\gamma}=2$.

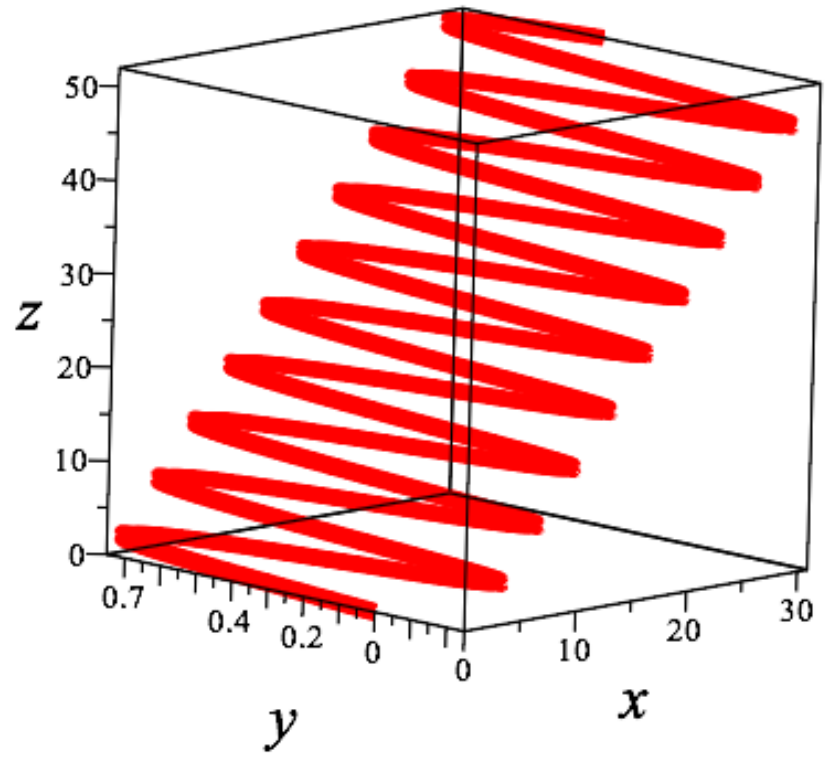

FIG. 3. Trajectory showing a cycloid in the absence of external torque (Eqs. (39)-(41)). Parameters are $\theta_{0}=\pi / 3, a_{0}=0.5, v_{s}=1$ and $\dot{\gamma}=2$.

Let us now return to the case $\lambda \neq 0$. For a general tumble trajectory a simple integration of (31) shows that the particle 
position is given by $\left(a=\lambda^{-2} \Gamma>1\right)$

$$
\begin{aligned}
x(t)=x(0) & +\left(\dot{\gamma} y(0)+\frac{v_{s}}{\tilde{\lambda}}-\frac{2 v_{s}}{\tilde{\lambda}} \ln (a)\right) t+\frac{2 v_{s}}{\tilde{\lambda}} \int_{0}^{t} \ln \left(a+\sin \left(\frac{\dot{\gamma} \Lambda}{2} s\right)\right) d s \\
& -\frac{4 a}{\sqrt{a^{2}-1}} \frac{v_{s} \Lambda}{\lambda}\left\{\tan ^{-1}\left(\frac{1}{\sqrt{a^{2}-1}}(1+a \tan (\dot{\gamma} \Lambda / 4 t))-\tan ^{-1}\left(\frac{1}{\sqrt{a^{2}-1}}\right)\right\},\right. \\
y(t)=y(0) & +\frac{2 v_{s}}{\lambda} \ln \left(\frac{a+\sin \left(\frac{\dot{\gamma} \Lambda}{2}\right) t}{a}\right) .
\end{aligned}
$$

The $z$-component is given by

$$
z(t)=z(0)+4 \frac{v_{s}}{\lambda} \frac{\sqrt{\tilde{\lambda}^{2} a^{2}-1}}{\sqrt{a^{2}-1}}\left\{\tan ^{-1}\left(\frac{1}{\sqrt{a^{2}-1}}(1+a \tan ((\dot{\gamma} \Lambda / 4) t))-\tan ^{-1}\left(\frac{1}{\sqrt{a^{2}-1}}\right)\right\} .\right.
$$

This result shows, as expected, that components $y$ and $z$ describe periodic curves, while component $x$ is unbounded with time. However, in the moving frame along $x$ with velocity $\left(\dot{\gamma} y(0)+\frac{v_{s}}{\grave{\lambda}}-\frac{2 v_{s}}{\tilde{\lambda}} \ln (a)\right) t$, the trajectory is periodic in the 3 directions (a closed trajectory). The trajectory is helical in the laboratory frame with a certain angle with respect to Cartesian axes (Fig. 4).

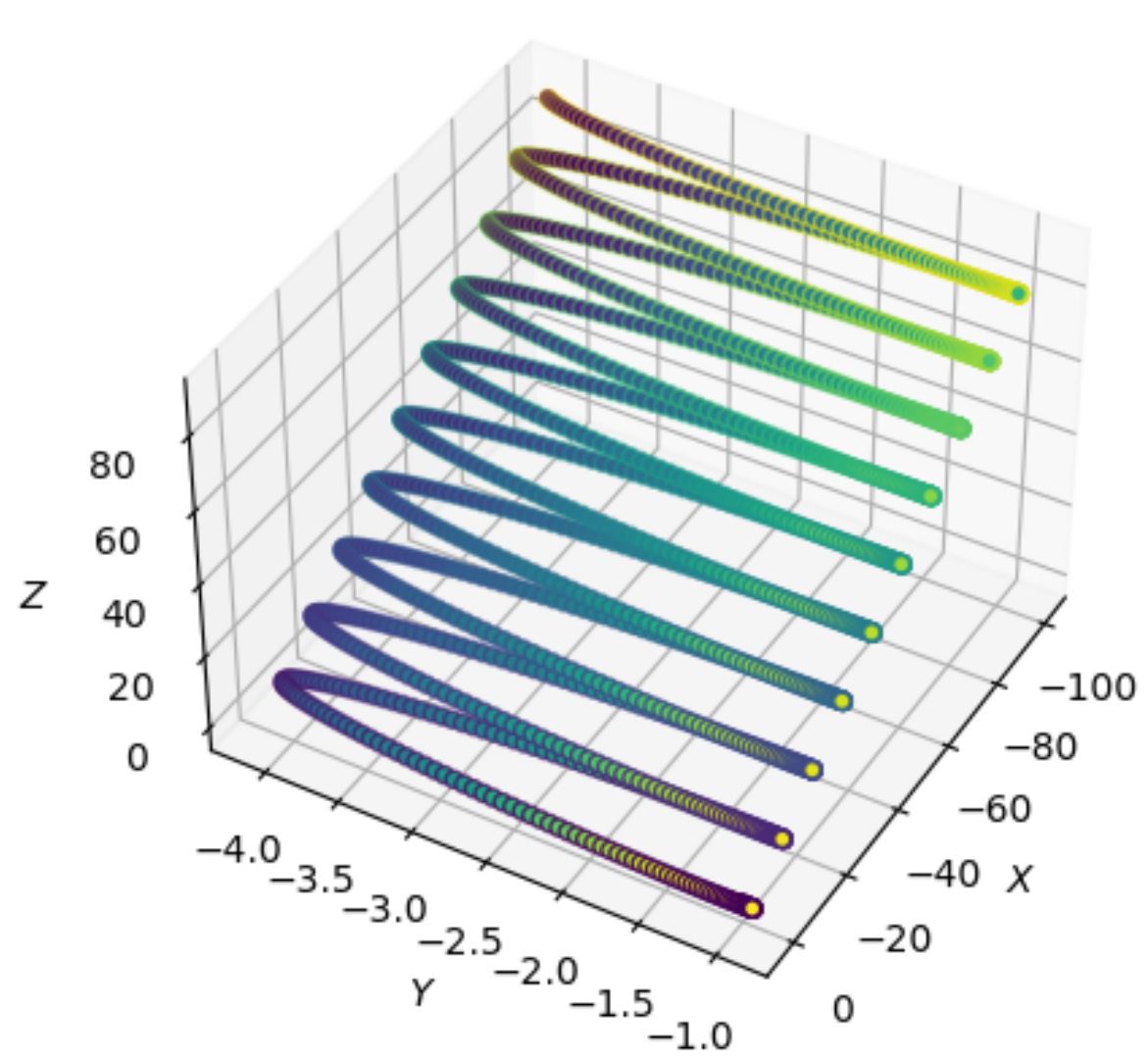

FIG. 4. Trajectory showing a helical shape. $\tilde{\lambda}=0.6, v_{s}=1, \dot{\gamma}=1, \Gamma=0.8$. 
To sum up the main results of this section, we have presented a simple method to analytically solve the (minimal) model that describes the motions of a spherical rigid sphere under flow and an external field. We have been able to derive a closed form of trajectories for the run and tumble regimes. In particular, it is found that the $x-y$ projection of the run trajectory describes a parabola, while in the tumbling regime the trajectory $y$ component oscillates and $x$ component increases with time; the trajectory is a helix. This result suggests, for example, that magnetotaxis might provide a method for identifying or separating rigid spherical magnetic swimmers as a function of their radii. More precisely, the swimmer exhibits a parabolic trajectory in the shear plane provided its radius (arising from $\tilde{\lambda}=\lambda / \dot{\gamma}>1$; run regime; see Eq. (6) for definition of $\lambda$ ) satisfies $r<\mathscr{M} h / 4 \pi \mu|\dot{\gamma}|=r_{c}$, while if $r>r_{c}(\tilde{\lambda}=\lambda / \dot{\gamma}>1)$ its trajectory is helical. Note that critical radius $r_{c}$ is determined by a competition between the flow and the external field (e.g. magnetic, gravitational).

\section{2D-BIDIRECTIONAL FLOWS}

In this Section, we consider the following general velocity field

$$
\mathbf{u}_{0}=\left(\delta x+\dot{\gamma}_{1} y, \dot{\gamma}_{2} x-\delta y, 0\right)
$$

where $\delta, \dot{\gamma}_{1}$ and $\dot{\gamma}_{2}$ are arbitrary constants. The above velocity field representation can be motivated by the fact that, in many cases, on the particle scale, the flow field may be linearized. We consider here three cases: a) external force is orthogonal to vorticity, b) external force is parallel to vorticity, and c) external force is neither orthogonal nor parallel to vorticity. We will consider gravity force and Coriolis force [17, 29, 30].

Let us note that since the (local) fluid vorticity is given by $\boldsymbol{\omega}=-\dot{\gamma} \boldsymbol{k}$, where $\dot{\gamma}=\dot{\gamma}_{1}-\dot{\gamma}_{2}$, we may assume that $\delta=0$ (since only $\boldsymbol{\omega}$ enters the $\boldsymbol{p}$ equation), i.e.,

$$
\mathbf{u}_{0}=\left(\dot{\gamma}_{1} y, \dot{\gamma}_{2} x, 0\right)
$$

where $\dot{\gamma}_{1} \dot{\gamma}_{2} \neq 0$. The opposite case where $\delta \neq 0$ and $\dot{\gamma}_{1}=\dot{\gamma}_{2}=0$, which corresponds to a stagnation flow, is treated in Section V.C. Since vorticity is along $\boldsymbol{k}$, the solutions for orientation vector $\boldsymbol{p}$ obtained for a simple shear flow remain valid.

\section{A. Vorticity and external force are perpendicular}

Let us consider first the effect of gravity $\boldsymbol{g}$ and take it to be parallel to $\boldsymbol{j}, \boldsymbol{g}=g \boldsymbol{j}$. The orientation $\boldsymbol{p}$ satisfies Eq. (5) with $\boldsymbol{j}^{\star}=\boldsymbol{j}, \omega_{1}=\omega_{2}=0, \omega_{3}=-\dot{\gamma}$, and parameter $\lambda=m g h / 4 \pi \mu r^{3}$, see (7). According to (25), the stable equilibrium orientation (if $|\dot{\gamma}|<|\lambda|)$ is given by

$$
p_{\text {eq }}=\frac{\dot{\gamma}}{\lambda} i+\frac{1}{\lambda} \sqrt{\lambda^{2}-\dot{\gamma}^{2}} \boldsymbol{j}
$$

As we have seen the particle tumbles if $|\dot{\gamma}|>|\lambda|$ and its orientation is given by solution (24). The particle trajectory is obtained by solving the following system

$$
\frac{d x}{d t}=\dot{\gamma}_{1} y+v_{s} \boldsymbol{p}_{x}, \quad \frac{d y}{d t}=\dot{\gamma}_{2} x+v_{s} \boldsymbol{p}_{y}, \quad \frac{d z}{d t}=v_{s} \boldsymbol{p}_{z} .
$$

Note that the flow can also be defined by a stream function $\Psi$ as

$$
\Psi(x, y)=\frac{1}{2}\left(\dot{\gamma}_{1} y^{2}-\dot{\gamma}_{2} x^{2}\right) .
$$

\section{Run trajectory}

We suppose that $|\tilde{\lambda}|>1$ ( we recall that $\tilde{\lambda}=\lambda / \dot{\gamma}$ ), and that the swimmer is at the stable equilibrium orientation (46). Therefore, the position $\mathbf{x}=(x, y, z)$ is governed by the following linear system

$$
\frac{d x}{d t}=\dot{\gamma}_{1} y+v_{s} \tilde{\lambda}^{-1}, \quad \frac{d y}{d t}=\dot{\gamma}_{2} x+v_{s} \sqrt{1-\tilde{\lambda}^{-2}}, \quad \frac{d z}{d t}=0 .
$$

Two different trajectories are obtained depending on the sign of $\dot{\gamma}_{1} \dot{\gamma}_{2}$. 
(a) For $\dot{\gamma}_{1} \dot{\gamma}_{2}>0$, the general solution has the following expression

$$
x=a e^{\sqrt{\dot{\gamma}_{1} \dot{\gamma}_{2}} t}+b e^{-\sqrt{\dot{\gamma}_{1} \dot{\gamma}_{2}} t}-\frac{v_{s}}{\dot{\gamma}_{2}} \sqrt{1-\tilde{\lambda}^{-2}},
$$

and

$$
y=a \frac{1}{\dot{\gamma}_{1}} \sqrt{\dot{\gamma}_{1} \dot{\gamma}_{2}} e^{\sqrt{\dot{\gamma}_{1} \dot{\gamma}_{2}} t}-b \frac{1}{\dot{\gamma}_{1}} \sqrt{\dot{\gamma}_{1} \dot{\gamma}_{2}} e^{-\sqrt{\dot{\gamma}_{1} \dot{\gamma}_{2}} t}-\frac{v_{s}}{\dot{\gamma}_{1}} \tilde{\lambda}^{-1}, \quad z=z(0),
$$

where $a$ and $b$ are arbitrary constants. Clearly, if $a=0, x$ and $y$ tend to $-\frac{v_{s}}{\dot{\gamma}_{2}} \sqrt{1-\tilde{\lambda}^{-2}}$ and $-\frac{v_{s}}{\dot{\gamma}_{1}} \tilde{\lambda}^{-1}$, respectively, while for $a \neq 0$, the $x-y$ plane projection long-time behavior is given by

$$
y \sim \frac{1}{\dot{\gamma}_{1}} \sqrt{\dot{\gamma}_{1} \dot{\gamma}_{2}} x
$$

or equivalently

$$
\dot{\gamma}_{2} x^{2} \sim \dot{\gamma}_{1} y^{2}
$$

A similar result can be found in [20] in which it is shown that the $x-y$ trajectories are hyperbolae. In fact, it is easily seen from (50) and (51) that $x$ and $y$ satisfy the following equation

$$
\left(x+\frac{v_{s}}{\dot{\gamma}_{2}} \sqrt{1-\tilde{\lambda}^{-2}}\right)^{2}-\left(\frac{\dot{\gamma}_{1}}{\sqrt{\dot{\gamma}_{1} \dot{\gamma}_{2}}} y+\frac{v_{s}}{\sqrt{\dot{\gamma}_{1} \dot{\gamma}_{2}}} \tilde{\lambda}^{-1}\right)^{2}=4 a b,
$$

confirming that trajectories are hyperbolae for any time (see Figure 5). This result shows that run trajectories follow the streamlines (see (48)).

(b) In the second case we suppose that $\dot{\gamma}_{2} \cdot \dot{\gamma}_{1}<0$ and that the particle is at the equilibrium orientation (as in the first case). Note that the above linear system (49) still holds. Exact analytical expressions of $\mathbf{x}=(x, y, z)$ can easily be obtained upon integration, yielding for some real parameters $a$ and $\theta_{0}$

$$
\begin{aligned}
& x=a \cos \left(\sqrt{\left|\dot{\gamma}_{1} \dot{\gamma}_{2}\right|} t-\theta_{0}\right)-\frac{v_{s}}{\dot{\gamma}_{2}} \sqrt{1-\tilde{\lambda}^{-2}}, \\
& y=-\frac{\sqrt{\left|\dot{\gamma}_{1} \dot{\gamma}_{2}\right|}}{\dot{\gamma}_{1}} a \sin \left(\sqrt{\left|\dot{\gamma}_{1} \dot{\gamma}_{2}\right|} t-\theta_{0}\right)-\frac{v_{s}}{\dot{\gamma}_{1}} \tilde{\lambda}^{-1}, \\
& z=z(0) .
\end{aligned}
$$

As a result, the $x-y$ projection of the trajectory has the form

$$
\left(x+\frac{v_{s}}{\dot{\gamma}_{2}} \sqrt{1-\tilde{\lambda}^{-2}}\right)^{2}+\left(\frac{\dot{\gamma}_{1}}{\sqrt{\left|\dot{\gamma}_{1} \dot{\gamma}_{2}\right|}} y+\frac{v_{s}}{\sqrt{\left|\dot{\gamma}_{1} \dot{\gamma}_{2}\right|}} \tilde{\lambda}^{-1}\right)^{2}=a^{2}
$$

and so, trajectories are ellipses constrained to a plane parallel to $x-y$ plane $(z=z(0))$ (see Figure 5). As in item (a) of the present Section, the trajectories follow the streamlines.

Note that, as a corollary of the above results, if we consider the following linear external flow [31]

$$
\mathbf{u}_{0}=s(x \boldsymbol{j}+y \boldsymbol{i})+\omega_{0}(x \boldsymbol{j}-y \boldsymbol{i}),
$$

in which $s$ is a measure of strength of the elongation flow component and $2 \omega_{0} \boldsymbol{k}$ is the vorticity vector, one sees, that for $\left|\omega_{0}\right|$ sufficiently small, we have elliptical trajectories if $|s|<\left|\omega_{0}\right|$, while in the opposite case trajectories are hyperbolae.

\section{Tumble trajectory}

We consider the case where $\dot{\gamma}_{1}=-\dot{\gamma}_{2}>0$ (the case $\dot{\gamma}_{1} \neq-\dot{\gamma}_{2}$ did not allow extraction of exact solution for trajectory). The tumbling regime corresponds to $|\tilde{\lambda}|<1$. We easily obtain the trajectory for $\mathbf{x}^{\prime}=(x, y)$ (the $x-y$ plane projection) by introducing $\mathbf{x}_{c}^{\prime}=x+i y$ (in complex notation), which satisfies (according to Eq. (3))

$$
\frac{d}{d t}\left(e^{i \dot{\gamma}_{1} t} \mathbf{x}_{c}^{\prime}\right)=v_{s} e^{i \dot{\gamma}_{1} t}\left(\boldsymbol{p}_{x}+i \boldsymbol{p}_{y}\right)
$$




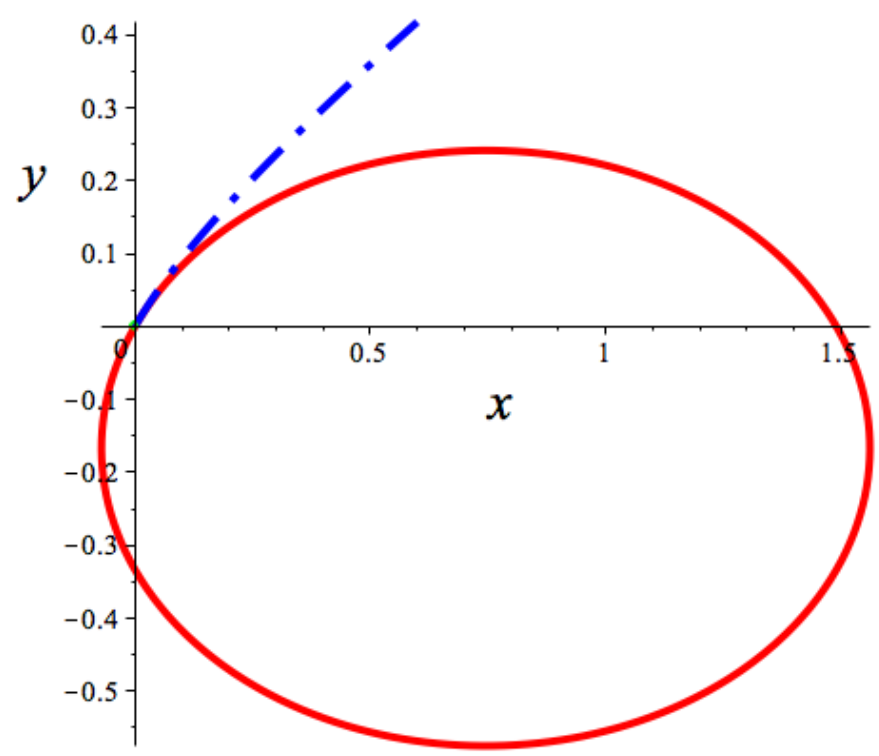

FIG. 5. Run trajectories when vorticity and external torques (gravity) are perpendicular. The fluid velocity is given by (45). Parameters are $\tilde{\lambda}=1.5, \dot{\gamma}_{1}=2$ and $v_{s}=0.5$. For the parabola (dashed-dotted blue line) $\dot{\gamma}_{2}=0.5$ and for the ellipse (solid red line) $\dot{\gamma}_{2}=-0.5$. For both trajectories the initial condition is $x(0)=y(0)=0$.

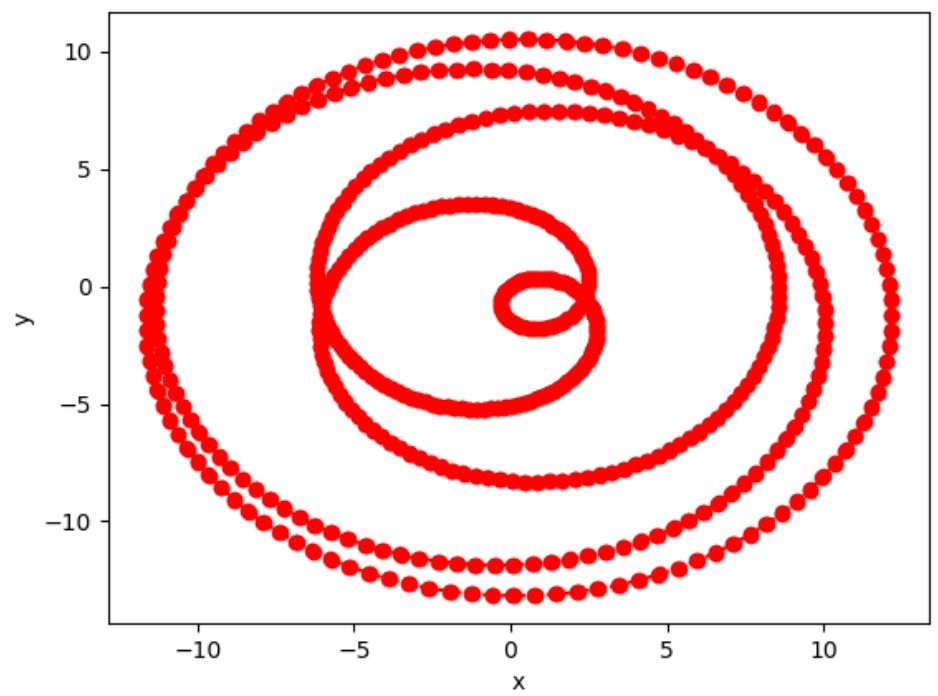

FIG. 6. A tumble trajectory in the $x-y$ plane according to (59) with $\mathbf{x}_{c}^{\prime}(0)=0$. Parameters are $\tilde{\lambda}=0.6, v_{s}=4, \dot{\gamma}=1, \dot{\gamma}_{1}=0.5$, and $a=3$.

Then, the expression of $\mathbf{x}_{c}^{\prime}$ can be rewritten as (solution for $\boldsymbol{p}$ remains formally identical as for a simple shear flow)

$$
\mathbf{x}_{c}^{\prime}=e^{-i \dot{\gamma}_{1} t} \mathbf{x}_{c}^{\prime}(0)+v_{s} e^{-i \dot{\gamma}_{1} t} \int_{0}^{t} e^{i \dot{\gamma}_{1} s}\left(\frac{\lambda^{-1}\left[\Gamma+\sin \left(\Lambda \dot{\gamma}_{1} s\right)\right]+i \tilde{\lambda}^{-1} \Lambda \cos \left(\Lambda \dot{\gamma}_{1} s\right)}{a+\sin \left(\Lambda \dot{\gamma}_{1} s\right)}\right) d s .
$$

Equation (59) shows that $\mathbf{x}_{c}^{\prime}$ or $(x, y)$ describes periodic trajectories (see Figure 6). The $z$-component of the particle position, which is periodic in time, is given by (43). The present findings agree with the conclusion of [20], obtained by numerical simulations. The trajectory is a sort of spherical helix (Fig. 7). 


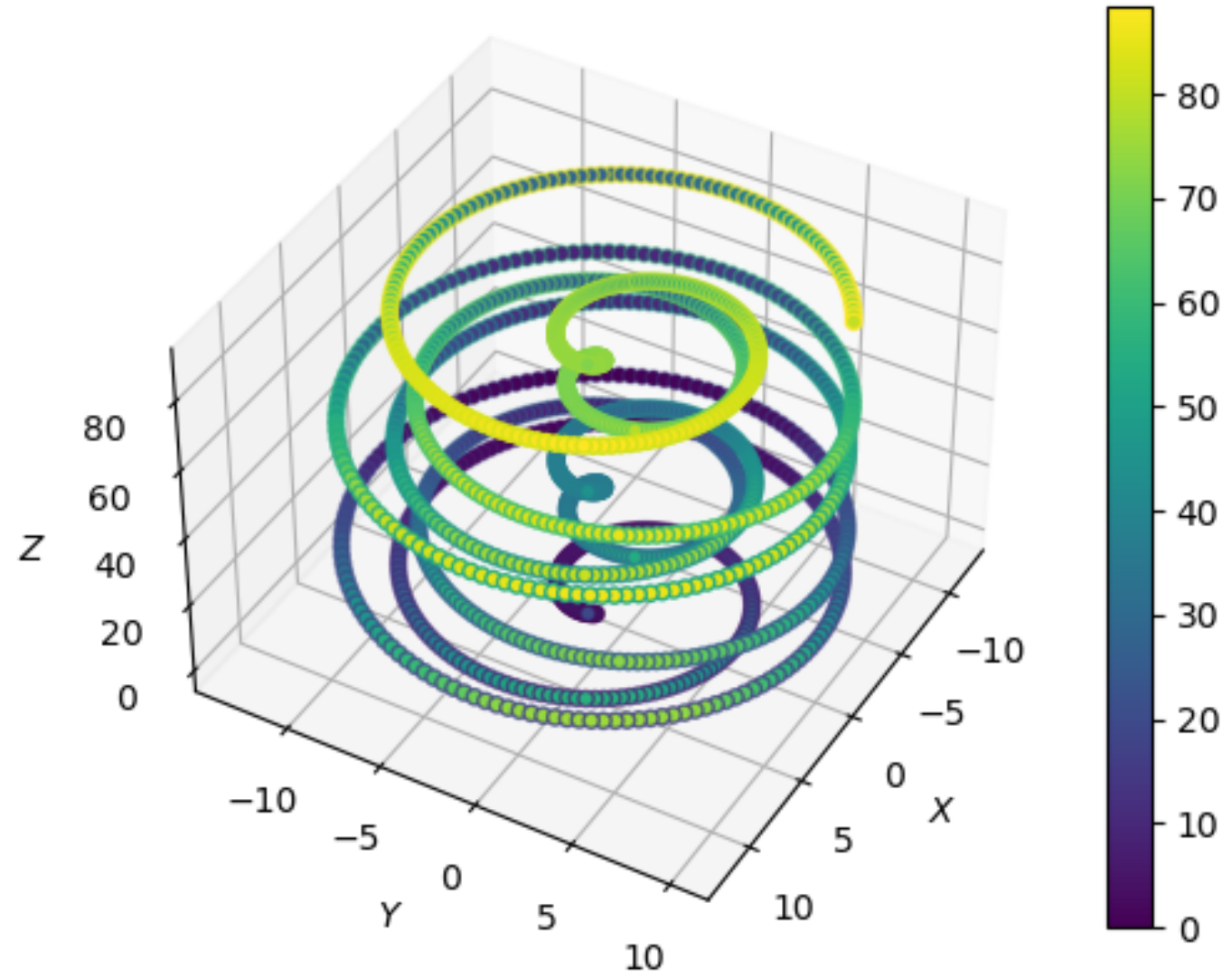

FIG. 7. Trajectory showing a helical shape. $\tilde{\lambda}=0.6, v_{s}=1, \dot{\gamma}=1, \Gamma=0.8$.

\section{B. External force parallel to vorticity}

Here we consider the case where gravity is parallel to vorticity. This is a simple and interesting example in which the orthogonality (A13) condition is not satisfied. As found previously [6,7], [8], in this case the swimmer orientation evolves in a run regime. However, we cannot use our approach to solve the equation of the orientation $\boldsymbol{p}$ in a closed form. We then restart from the evolution equation itself and it turns out that the solution is simply obtained in this case.

By assuming $\boldsymbol{g}=-g \boldsymbol{k}$, orientation $\boldsymbol{p}$ satisfies

$$
\left\{\begin{array}{l}
2 \frac{d p_{x}}{d t}=\dot{\gamma} p_{y}+\lambda p_{z} p_{x} \\
2 \frac{d p_{y}}{d t}=-\dot{\gamma} p_{x}+\lambda p_{z} p_{y} \\
2 \frac{d p_{z}}{d t}=\lambda\left[p_{z}^{2}-1\right]
\end{array}\right.
$$

The above system has exactly two stationary solutions $\boldsymbol{p}= \pm \boldsymbol{k}$ and only equilibrium $-\boldsymbol{k}$ is stable.

In this case only run trajectory exists, and the trajectory is obtained by solving

$$
\frac{d x}{d t}=\dot{\gamma}_{1} y, \quad \frac{d y}{d t}=\dot{\gamma}_{2} x, \quad \frac{d z}{d t}=-v_{s} .
$$


Here, it is assumed that the swimmer is at the stable equilibrium orientation $(\boldsymbol{p}=-\boldsymbol{k})$.

As above, two different run trajectories are obtained depending on the sign of $\dot{\gamma}_{1} \dot{\gamma}_{2}$.

1. For $\dot{\gamma}_{1} \dot{\gamma}_{2}>0$, we have

$$
\begin{gathered}
x=a e^{\sqrt{\dot{\gamma}_{1} \dot{\gamma}_{2}} t}+b e^{-\sqrt{\dot{\gamma}_{1} \dot{\gamma}_{2}} t}, \\
y=a \frac{1}{\dot{\gamma}_{1}} \sqrt{\dot{\gamma}_{1} \dot{\gamma}_{2}} e^{\sqrt{\dot{\gamma}_{1} \dot{\gamma}_{2}} t}-b \frac{1}{\dot{\gamma}_{1}} \sqrt{\dot{\gamma}_{1} \dot{\gamma}_{2}} e^{-\sqrt{\dot{\gamma}_{1} \dot{\gamma}_{2}} t},
\end{gathered}
$$

and

$$
z=z(0)-v_{s} t
$$

where $a$ and $b$ are arbitrary constants.

2. For $\dot{\gamma}_{2} \cdot \dot{\gamma}_{1}<0$, the particle trajectory satisfies, for real parameters $a$ and $\theta_{0}$,

$$
\begin{aligned}
& x=a \cos \left(\sqrt{\left|\dot{\gamma}_{1} \dot{\gamma}_{2}\right|} t-\theta_{0}\right), \\
& y=-\frac{\sqrt{\left|\dot{\gamma}_{1} \dot{\gamma}_{2}\right|}}{\dot{\gamma}_{1}} a \sin \left(\sqrt{\left|\dot{\gamma}_{1} \dot{\gamma}_{2}\right|} t-\theta_{0}\right), \\
& z=z(0)-v_{s} t .
\end{aligned}
$$

Let us note, by making use of the above exact solutions for system (61), that components $x$ and $y$ satisfy

$$
\dot{\gamma}_{1} x^{2}-\dot{\gamma}_{2} y^{2}=\text { const }
$$

This shows that the $x-y$ trajectories are hyperbolae for $\dot{\gamma}_{1} \cdot \dot{\gamma}_{2}>0$, while for $\dot{\gamma}_{1} \dot{\gamma}_{2}<0$ the swimmer trajectory describes an elliptical cylinder.

\section{Solid body rotation with external force having a general orientation with respect to vorticity}

Here, we study the orientation dynamics and the trajectories of spherical gyrotactic particles subject to a solid body rotation $\left(\delta=0, \dot{\gamma}_{1}=-\dot{\gamma}_{2}=-\dot{\gamma}\right)$

$$
\mathbf{u}_{0}=-\dot{\gamma}(y \dot{i}-x \dot{j})
$$

which has the property that the vorticity, which is parallel to $z$-axis, has constant angular velocity; $\boldsymbol{\omega}=\nabla \times \mathbf{u}_{0}=2 \dot{\gamma} \boldsymbol{k}$. Here, we assume that gravity is parallel to vorticity; $\boldsymbol{g}=-\boldsymbol{g} \boldsymbol{k}$. This section is motivated by a (deterministic) model given in [17, 29, 30] for which the equation of the swimming direction is rewritten (following their notation for the sake of comparison) as

$$
\dot{\boldsymbol{p}}=-\frac{1}{2 v_{0}}[\boldsymbol{A}-\boldsymbol{A} \cdot \boldsymbol{p} \boldsymbol{p}]+\frac{1}{2} \boldsymbol{\omega} \times \boldsymbol{p}
$$

where $v_{0}=3 \mu / h, h$ is the cell center-of-mass displacement, $\mu$ fluid kinematic viscosity, and (in the presence of fluid acceleration) $\boldsymbol{A}$ is the total acceleration which is given by $[17,29,30,32]$

$$
\boldsymbol{A}=\boldsymbol{g}-\boldsymbol{a}
$$

where the fluid acceleration $\boldsymbol{a}=\left(-\dot{\gamma}^{2} x,-\dot{\gamma}^{2} y, 0\right)$. This is the Coriolis force due to cylinder rotation[17, 29, 30]. Equation (68) is used in [18] to study the effect of the fluid acceleration on spherical gyrotactic microorganisms orientation. Note that unlike $\boldsymbol{j}^{\star}$ in Eq. (5), $\boldsymbol{A}$ is not normed to unity. Here, we shall first investigate in detail two limiting cases; $\boldsymbol{A}=\boldsymbol{g}$, and $\boldsymbol{A}=-\boldsymbol{a}$. The generalized case $\boldsymbol{A}=\boldsymbol{g}-\boldsymbol{a}$, which seems to be not amenable to exact analytical solution, will be discussed in the last Subsection. Note that in this case the total acceleration $\boldsymbol{A}$ is neither orthogonal nor parallel to fluid vorticity ( and the orthogonality condition on $\boldsymbol{j}^{\star}$ and $\boldsymbol{p}_{0}$ is not fulfilled so that solution (15) cannot be used). This means that solution (A15) does not apply, and we shall use a different approach. 


\section{Effect of the gravity}

In the first limit $\boldsymbol{A}=\boldsymbol{g}$, the external field and the fluid vorticity are parallel. In a such situation, it is known that the orientation vector tends to a certain fixed orientation (see Refs. [6-8]). Comparing the swimming direction equations given by (68) and (5), we have $\boldsymbol{j}^{\star}=\boldsymbol{k}, \lambda=g / v_{0}, \omega_{1}=\omega_{2}=0$ and $\omega_{3}=2 \dot{\gamma}$. Since here $\boldsymbol{g}$ and $\boldsymbol{\omega}$ are not orthogonal, the fixed orientation solution (A15) is not valid, and one has thus to go back to the original equation, which turns out to have a simple solution. In terms of the Cartesian coordinates of $\boldsymbol{p}$, Eq. (68) reads

$$
\left\{\begin{array}{l}
\frac{d p_{x}}{d t}=\dot{\gamma}\left[-p_{y}-\tilde{\lambda}_{g} p_{z} p_{x}\right], \\
\frac{d p_{y}}{d t}=\dot{\gamma}\left[p_{x}-\tilde{\lambda}_{g} p_{z} p_{y}\right], \\
\frac{d p_{z}}{d t}=\dot{\gamma}\left[\tilde{\lambda}_{g}-\tilde{\lambda}_{g} p_{z}^{2}\right],
\end{array}\right.
$$

where

$$
\tilde{\lambda}_{g}=\frac{g}{2 v_{0} \dot{\gamma}} .
$$

Note that $\boldsymbol{p}= \pm \boldsymbol{k}$ are the (only) two exact stationary solutions for all values of parameter $\tilde{\lambda}_{g}$.

Solving first the $p_{z}$ equation and substituting it into the first and second equations of (70) yields

$$
\begin{aligned}
& p_{x}=2 \frac{p_{x}(0) \cos (\dot{\gamma} t)-p_{y}(0) \sin (\dot{\gamma} t)}{\left(1+p_{z}(0)\right) e^{\dot{\gamma} \tilde{\lambda}_{g} t}+\left(1-p_{z}(0)\right) e^{-\dot{\gamma} \tilde{n}_{g} t}}, \\
& p_{y}=2 \frac{p_{x}(0) \sin (\dot{\gamma} t)+p_{y}(0) \cos (\dot{\gamma} t)}{\left(1+p_{z}(0)\right) e^{\dot{\gamma} \tilde{\lambda}_{g} t}+\left(1-p_{z}(0)\right) e^{-\dot{\gamma} \tilde{\lambda}_{g} t}},
\end{aligned}
$$

and

$$
p_{z}(t)=\frac{\left(1+p_{z}(0)\right) e^{\dot{\gamma} \tilde{\lambda}_{g} t}-\left(1-p_{z}(0)\right) e^{-\dot{\gamma} \tilde{\lambda}_{g} t}}{\left(1+p_{z}(0)\right) e^{\dot{\gamma} \tilde{\lambda}_{g} t}+\left(1-p_{z}(0)\right) e^{-\dot{\gamma} \tilde{\lambda}_{g} t}}
$$

with $p_{x}^{2}(0)+p_{y}^{2}(0)+p_{z}^{2}(0)=1$.

Thus, we see that, as $t \rightarrow \infty, \boldsymbol{p}$ tends to the (only) stable steady solution $\boldsymbol{p}_{\text {eq }}=\boldsymbol{k}$ (recall that $\dot{\gamma} \tilde{\lambda}>0$ ). This shows that the gyrotactic particle exhibits a run motion and aligns in the direction opposite to gravity for large $t$.

Expressions (72) and (73) are obtained by introducing $q=p_{x}+i p_{y}$, as in the proof of (59). It is easily found that

$$
q=2 q(0) \frac{e^{i \dot{\gamma} t}}{\left(1+p_{z}(0)\right) e^{i \tilde{\lambda}_{g} t}+\left(1-p_{z}(0)\right) e^{-\dot{\gamma} \tilde{\lambda}_{g} t}}
$$

This result can also be deduced by introducing $\rho=\sqrt{p_{x}^{2}+p_{y}^{2}}$, which satisfies the simple ordinary differential equation

$$
\frac{d \rho}{d t}=-\dot{\gamma} \tilde{\lambda}_{g} p_{z} \rho
$$

For the particle position, it is convenient to solve the following system

$$
\frac{d \mathbf{x}_{c}}{d t}=\dot{\gamma} i \mathbf{x}_{c}+v_{s} q
$$

and

$$
\frac{d z}{d t}=v_{s} p_{z}
$$


where $\mathbf{x}_{c}=x+i y$ and $q$ is given by Eq. (75). Therefore,

$$
\mathbf{x}_{c}=e^{i \dot{\gamma} t}\left[\mathbf{x}_{c}(0)+v_{s} e^{i \theta_{0}} \int_{0}^{t} \rho(s) d s\right]
$$

and

$$
z=z(0)+\frac{v_{s}}{\dot{\gamma} \tilde{\lambda}_{g}} \ln \frac{H(t)}{2}
$$

where $\theta_{0}$ is the initial angle of $q$ (i.e., $q(0)=\rho(0) e^{i \theta_{0}}$ ) and

$$
H(t)=\left(1+p_{z}(0)\right) e^{\dot{\gamma} \tilde{\lambda}_{g} t}+\left(1-p_{z}(0)\right) e^{-\dot{\gamma} \tilde{\lambda}_{g} t} .
$$

Note that if $p_{z}(0)= \pm 1$ (this holds (only) if the particle is at the stable or unstable equilibrium orientation), we easily deduce that the full $3 \mathrm{D}$ trajectory is given by

$$
\mathbf{x}_{c}=e^{i \dot{\gamma} t} \mathbf{x}_{c}(0), \quad z=z(0) \pm v_{s} t
$$

which describes exactly a helical trajectory in the positive $z$-direction for $p_{z}(0)=1$ at the speed $v_{s}$. Each particle exhibits a helical trajectory with radius $\left|\mathbf{x}_{c}(0)\right|$. This means that a homogeneous dilute suspension (i.e. particle-particle interaction is negligible) remains homogeneous where each particle rotates around z-axis following a helical path with a radius given by the initial position.

Next, we assume that $p_{z}(0) \neq \pm 1$. By making use of the exact expression of $\rho$, one sees that $\mathbf{x}_{c}$ can be written as,

$$
\mathbf{x}_{c}=e^{i \dot{\gamma} t}\left[\mathbf{x}_{c}(0)+e^{i \theta_{0}} \frac{2 v_{s}}{\dot{\gamma} \tilde{\lambda}_{g}}\left(\arctan \left(d e^{\dot{\gamma} \tilde{\lambda}_{g} t}\right)-\arctan d\right)\right],
$$

or, equivalently for $0<d<\infty$,

$$
\mathbf{x}_{c}=e^{i \dot{\gamma} t}\left[\mathbf{x}_{c}(0)+e^{i \theta_{0}} \frac{2 v_{s}}{\dot{\gamma} \tilde{\lambda}_{g}}\left(\arctan \left(d^{-1}\right)-\arctan d^{-1} e^{-\dot{\gamma} \tilde{\lambda}_{g} t}\right)\right],
$$

where $d=\sqrt{\left(1+p_{z}(0)\right) /\left(1-p_{z}(0)\right)}$. Equations (80) and (83) or (84) define the trajectory of a spherical gyrotactic particle in rotating fluid.

Note that parameter $d$ can be written as

$$
d=\sqrt{\frac{1+\cos \left(\phi_{0}\right)}{1-\cos \left(\phi_{0}\right)}}
$$

where $0 \leq \phi_{0} \leq \pi$ is the angle between $\boldsymbol{k}$ and the initial orientation vector $\boldsymbol{p}(0)$.

Let us examine in detail the behavior of the trajectory for large $t$. The explicit exact solutions show that, for large $t$, trajectories converge exponentially to different helical trajectories, depending on initial conditions (initial position and orientation);

$$
e^{-i \dot{\gamma} t} \mathbf{x}_{c} \rightarrow \tilde{\mathbf{x}}_{d}(0)=\mathbf{x}_{c}(0)+e^{i \theta_{0}} \frac{2 v_{s}}{\dot{\gamma} \tilde{\lambda}_{g}} \arctan d^{-1}, \quad z(t) \rightarrow v_{s} t
$$

Note that quantity $e^{i \theta_{0}} 2 v_{s} / \dot{\gamma} \tilde{\lambda}_{g}$ arctan $d^{-1}$ depends only on initial orientation $\boldsymbol{p}(0)$.

As a way of example, let us assume that $\mathbf{x}_{c}(0)=0$. Rewriting Eq. (84) gives

$$
\left\{\begin{array}{l}
x(t)=\frac{2 v_{s}}{\dot{\gamma} \tilde{\lambda}_{g}}\left(\arctan d^{-1}-\arctan d^{-1} e^{-\dot{\gamma} \tilde{\lambda}_{g} t}\right) \cos \left(\dot{\gamma} t+\theta_{0}\right), \\
y(t)=\frac{2 v_{s}}{\dot{\gamma} \tilde{\lambda}_{g}}\left(\arctan d^{-1}-\arctan d^{-1} e^{-\tilde{\lambda}_{g} t}\right) \sin \left(\dot{\gamma} t+\theta_{0}\right) .
\end{array}\right.
$$


Recall that coordinate $z$ is given by (82). In terms of the dynamical system, we may conclude that there exists a family of trajectories represented by

$$
\mathscr{A}_{d}=\left(e^{i \dot{\gamma} t} \tilde{\mathbf{x}}_{d}(0), v_{s} t\right),
$$

in $\mathbb{C} \times \mathbb{R}$, that are possible attractor of the dynamics. For each $0<d<\infty$, the set $\mathscr{A}_{d}$ describes cylinder or helical (trajectory) whose radius, designated by $\rho_{d}$, is given by

$$
\rho_{d}=4 \frac{v_{s} v_{0}}{g} \arctan d^{-1} .
$$

Note that the $x-y$ projection of the trajectories follow the streamlines and that radius $\rho_{d}$ decreases from $2 \pi v_{s} v_{0} / g$

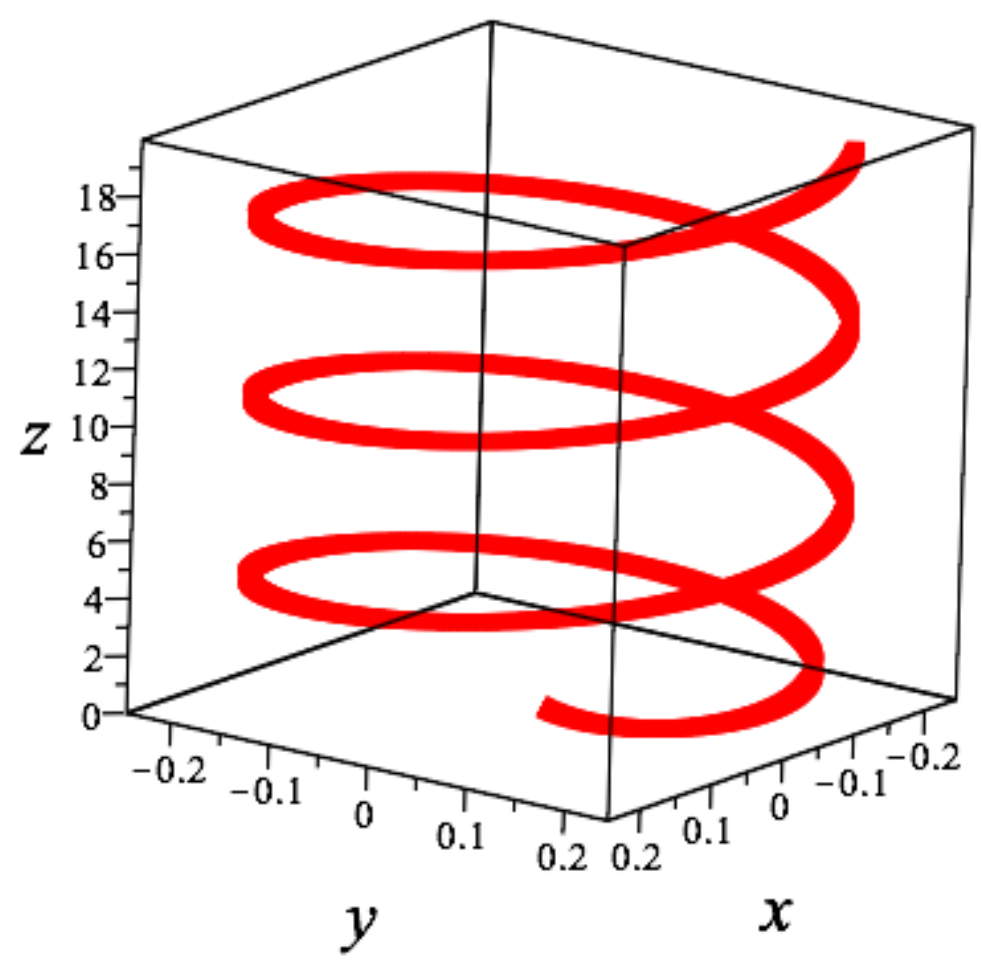

FIG. 8. An example of trajectories showing that the microswimmer approaches a pseudo-attractor. Parameters are $\lambda=1, \tilde{\lambda}_{g}=2, \dot{\gamma}=1, \theta_{0}=$ $\pi / 3, v_{s}=1$ and $d=4$.

(the radius of the upper helical) to 0 , as $d$ increases from 0 to $\infty$. In the limiting case $d=0$ (i.e., $\boldsymbol{p}=(0,0,-1)$, the unstable equilibrium orientation), or $d=\infty$ (i.e., $\boldsymbol{p}=(0,0,1)$, the stable equilibrium orientation), the particle moves along the $z$-axis at the velocity $\mp v_{s}$. For $0<d<\infty$, the trajectory is three-dimensional. The particle migrates to the helical trajectory (with radius satisfying (89)), which may play the same role as an attractor (see Figure 8).

$\mathscr{A}_{d}$ can be considered as a pseudo-attractor or "streamlining" attractor in the sense that for any initial position $\left(\mathbf{x}_{c}(0), z(0)\right) \in$ $\mathscr{A}_{d}$ such that $0<d<\infty$, (i. e., $x(0)^{2}+y^{2}(0) \neq 0$ ), the particle migrates from $\left(\mathbf{x}_{c}(0), z(0)\right)$ to $\tilde{\mathscr{A}}_{d}=\left(e^{i \dot{\gamma}_{t}} \tilde{\mathbf{x}}_{d}(0), v_{s} t\right)$. The final state depends on initial conditions, so that the final trajectory is not a limit cycle.

The above result suggests that two particles 1 and 2 such that $\tilde{\mathbf{x}}_{1 d}(0)=\tilde{\mathbf{x}}_{2 d}(0)$ will tend to approach the same helical trajectory for large time. Counterintuitively, we find that two particles with same initial position but with different initial orientations do not tend to the same trajectory. Thus, if initially particles are prepared with different orientations, they form different helical paths as time proceeds (see Figure 9).

In passing, we may observe from (85) that quantity $\rho_{d}$ reads simply

$$
\rho_{d}=2 \frac{v_{s} v_{0}}{g} \phi_{0}
$$

indicating that $\rho_{d}$ increases linearly with angle $\phi_{0}$. 


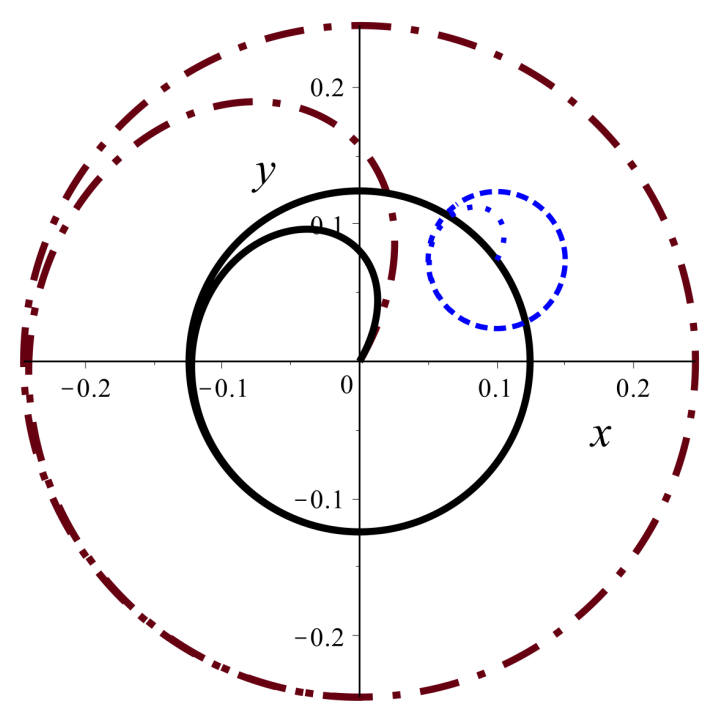

FIG. 9. Different $x-y$ trajectories of the microswimmer as a function of the initial position $(x(0), y(0))$ and the parameter $d$ or the initial orientation $\phi_{0}$. Here, parameters are $\lambda=1, \tilde{\lambda}_{g}=2, \dot{\gamma}=1, \theta_{0}=\pi / 3, v_{s}=1$ and $d=4$ (dotted-dashed brown line), $d=8$ (solid black line) and $d=20$ (dashed blue line). Trajectories show that the microswimmer approaches a circular path in $x-y$ plane.

\section{Effect of fluid acceleration}

Here, we briefly consider Eqs. (67) and (68) in the limiting case $\boldsymbol{A}=-\boldsymbol{a}=\left(\dot{\gamma}^{2} x, \dot{\gamma}^{2} y, 0\right)$ (gravity is neglected) which isolates the effect of fluid acceleration [17]. Vector $\boldsymbol{j}^{\star}$ is then given by

$$
j^{\star}=\left(\frac{x}{\sqrt{x^{2}+y^{2}}}, \frac{y}{\sqrt{x^{2}+y^{2}}}, 0\right)
$$

which is orthogonal to fluid vorticity $\boldsymbol{\omega}=2 \dot{\gamma} \boldsymbol{k}$. According to Section III, the swimmer exhibits a tumbling regime in region where $\dot{\gamma}^{2}\left(x^{2}+y^{2}\right)<4 v_{0}^{2}$, while in region $\dot{\gamma}^{2}\left(x^{2}+y^{2}\right)>4 v_{0}^{2}$, the particle swims in the stable equilibrium direction

$$
p_{\mathrm{eq}}=\frac{1}{\lambda} \frac{\lambda^{2} a^{2}-\omega_{3}^{2}}{b \omega_{3}+a \sqrt{\lambda^{2}-\omega_{3}^{2}}} i+\frac{1}{\lambda} \frac{\lambda^{2} a b+\omega_{3} \sqrt{\lambda^{2}-\omega_{3}^{2}}}{b \omega_{3}+a \sqrt{\lambda^{2}-\omega_{3}^{2}}} j
$$

where

$$
\omega_{3}=2 \dot{\gamma}, a=\frac{x}{\sqrt{x^{2}+y^{2}}}, b=\frac{y}{\sqrt{x^{2}+y^{2}}}, \lambda=-\frac{\dot{\gamma}^{2}}{v_{0}} \sqrt{x^{2}+y^{2}}
$$

We may then conclude that a gyrotactic swimmer in the considered fluid acceleration may accumulate towards center of cylinder. To show this we study the swimmer trajectory. Since the swimmer position satisfies $(z(t)=z(0))$

$$
\frac{d x}{d t}=-\dot{\gamma} y+\frac{v_{s}}{\lambda} \frac{\lambda^{2} a^{2}-\omega_{3}^{2}}{b \omega_{3}+a \sqrt{\lambda^{2}-\omega_{3}^{2}}}, \quad \frac{d y}{d t}=\dot{\gamma} x+\frac{v_{s}}{\lambda} \frac{\lambda^{2} a b+\omega_{3} \sqrt{\lambda^{2}-\omega_{3}^{2}}}{b \omega_{3}+a \sqrt{\lambda^{2}-\omega_{3}^{2}}},
$$

one deduces that the radial position, $\rho=\sqrt{x^{2}+y^{2}}$, evolves according to

$$
\rho \frac{d \rho}{d t}=-\frac{v_{0} v_{s}}{\dot{\gamma}^{2}} \sqrt{\frac{\dot{\gamma}^{4}}{v_{0}^{2}} \rho^{2}-\omega_{3}^{2}} .
$$

The solution of the above equation is

$$
\frac{\dot{\gamma}^{4}}{v_{0}^{2}} \rho^{2}=\omega_{3}^{2}+\left(\sqrt{\frac{\dot{\gamma}^{4}}{v_{0}^{2}} \rho^{2}(0)-\omega_{3}^{2}}-\frac{\dot{\gamma}^{2} v_{s}}{v_{0}} t\right)^{2} .
$$




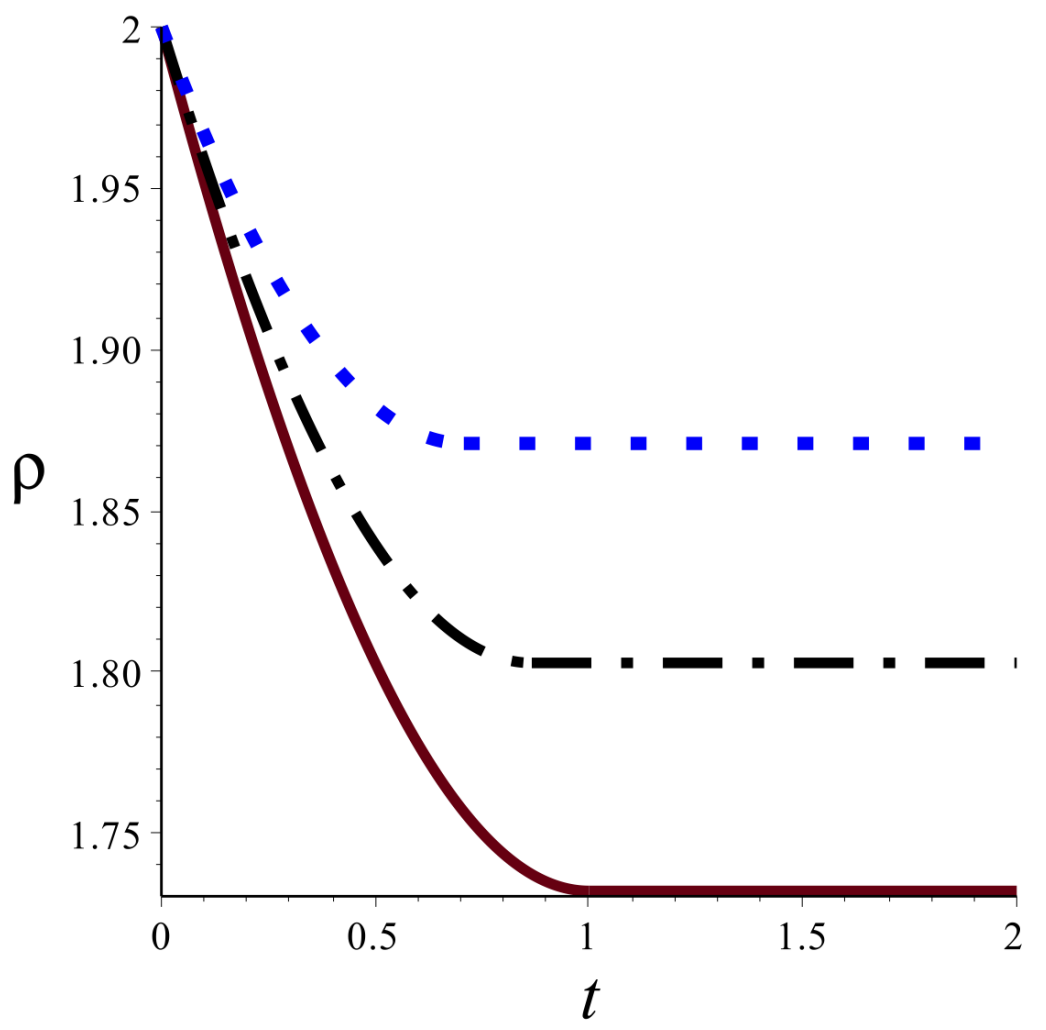

FIG. 10. Exact solutions to the ordinary differential equation (95) with $\dot{\gamma}^{2} / v_{0}=1, v_{s}=1, \rho(0)=2$ and for different values of $w_{3}$ ( $w_{3}^{2}=3$ for solid brown line, $w_{3}^{2}=3.25$ for dotted-dashed black line and $w_{3}^{2}=3.5$ for dotted blue line). $\rho$ reaches the fixed point $2 v_{0} / \dot{\gamma}$ at finite time, $t^{\star}$, and satisfies $\rho=2 v_{0} / \dot{\gamma}$ for all $t>t^{\star}$.

Solution (96) predicts that the radial distance of a swimmer located initially in the run region (i.e. $\left.\dot{\gamma}^{2}\left(x^{2}+y^{2}\right)>4 v_{0}^{2}\right)$ decreases as $t$ increases, and that the particle reaches the cylinder

$$
\mathscr{A}=\left\{\dot{\gamma}^{2}\left(x^{2}+y^{2}\right)=4 v_{0}^{2}\right\}
$$

at finite time $t^{\star}$;

$$
t^{\star}=\frac{v_{0}}{\dot{\gamma}^{2} v_{s}} \sqrt{\frac{\dot{\gamma}^{4}}{v_{0}^{2}} \rho^{2}(0)-\omega_{3}^{2}} .
$$

Let us recall that cylinder $\mathscr{A}$ separates tumble and run regions. In fact, we can see that any horizontal trajectory of $\mathscr{A}$ satisfies system (116). Hence, we may conclude that any run trajectory reaches cylinder $\mathscr{A}$ at a finite time and after this time the swimmer executes a circular orbit (around the rotation axis) that follows a streamline:

$$
x(t)=\frac{2 v_{0}}{\dot{\gamma}} \cos \left(\dot{\gamma}\left(-1+\frac{v_{s}}{2 v_{0}}\right) t+\theta_{0}\right), \quad y(t)=\frac{2 v_{0}}{\dot{\gamma}} \sin \left(\dot{\gamma}\left(-1+\frac{v_{s}}{2 v_{0}}\right) t+\theta_{0}\right),
$$

where $\theta_{0}$ is a constant. This result show that fluid acceleration may generate accumulations at finite time around cylinder of radius $2 v_{0} / \dot{\gamma}$. Time $t^{\star}$ can be referred to as the focusing time for a run trajectory (see Figure 10 ).

\section{Gyrotactic swimmer in a fluid acceleration field}

We consider here both gravity and fluid acceleration. In this case, the total force $\boldsymbol{A}$ is neither parallel nor perpendicular to the vorticity. As a preliminary step we investigate Eqs. (67)-(69) in the limit where the vorticity is relatively small. This leads to an approximate solution of trajectory equations; Eqs. (67)-(69). The solution of this particular case has been reported in [29]. By neglecting the vorticity the swimmer direction equation (Eq. (68)) becomes

$$
\dot{\boldsymbol{p}}=\frac{1}{2} \lambda(\boldsymbol{A}-\boldsymbol{A} \cdot \boldsymbol{p} \boldsymbol{p}),
$$


where $\lambda=-1 / v_{0}$ and (total acceleration) $\boldsymbol{A}=\left(\dot{\gamma}^{2} x, \dot{\gamma}^{2} y,-g\right)$. The above equation has one stable equilibrium swimming direction given by $\boldsymbol{p}_{\mathrm{eq}}^{\star}=-\boldsymbol{A} /\|\boldsymbol{A}\|$;

$$
\boldsymbol{p}_{\mathrm{eq}}^{\star}=\left(-\frac{x}{\sqrt{x^{2}+y^{2}+\kappa^{2}}},-\frac{y}{\sqrt{x^{2}+y^{2}+\kappa^{2}}}, \frac{\kappa}{\sqrt{x^{2}+y^{2}+\kappa^{2}}}\right),
$$

where $\kappa=g / \dot{\gamma}^{2}$. The equation of the radial swimmer position, $\rho^{2}=x^{2}+y^{2}$, can be written as

$$
\frac{d \rho}{d t}=-v_{s} \frac{\rho}{\sqrt{\rho^{2}+\kappa^{2}}}
$$

For the two limiting cases $\rho \ll \kappa$ and $\rho \gg \kappa$ the above ordinary differential equation becomes linear and we may deduce that $\rho$ decays exponentially if $\rho \ll \kappa$, while if $\rho \gg \kappa$ one sees that the radial swimmer position is given by $\rho(t)=$ $\rho(0)-v_{s} t$. This linear decrease can also be deduced from (96).

For arbitrary $\rho(0)$, a solution to Eq. (102) can be obtained by a simple integration. It is found that the radial swimmer position (for small vorticity) can be deduced from

$$
\sqrt{(\rho / \kappa)^{2}+1}=\sqrt{(\rho(0) / \kappa)^{2}+1}-\frac{v_{s}}{\kappa} t-\frac{1}{2} \ln \left(\frac{\mathscr{H}(t)}{\mathscr{H}(0)}\right),
$$

where

$$
\mathscr{H}(t)=\frac{\sqrt{(\rho / \kappa)^{2}+1}-1}{\sqrt{(\rho / \kappa)^{2}+1}+1} .
$$

It follows from (103) that the radial particle position behaves, for $t \rightarrow \infty$, as

$$
\rho(t) \sim 2 \kappa e^{-\frac{v_{S}}{\kappa} t}
$$

Let us now return to Eqs. (67)-(69) in which the swimmer is subjected to both gravitational and fluid acceleration. Let us recall that $\boldsymbol{A}$ is not orthogonal to the fluid vorticity, so that the swimmer direction will tend in time to a certain (stable) equilibrium orientation irrespective of its initial orientation. We will see that taking into account vorticity will lead to some difference with the above study.

Equation (68) is rewritten as

$$
\dot{\gamma}^{-1} \dot{\mathbf{p}}=\boldsymbol{k} \times \boldsymbol{p}+\lambda\left[\boldsymbol{j}^{\star}-\dot{j}^{\star} \cdot \boldsymbol{p p}\right]
$$

where $\boldsymbol{j}^{\star}=-\boldsymbol{A} /\|\boldsymbol{A}\| \equiv\left(j_{x}^{\star}, j_{y}^{\star}, j_{z}^{\star}\right)$ and

$$
\lambda=\lambda_{0} \sqrt{x^{2}+y^{2}+\kappa^{2}}, \quad \lambda_{0}=\frac{\dot{\gamma}}{2 v_{0}}
$$

By assuming $\dot{\boldsymbol{p}}=0$, the equation of the equilibrium swimming direction is then

$$
\left\{\begin{array}{rr}
0= & -p_{y}+\lambda j_{x}^{\star}-\lambda \boldsymbol{j}^{\star} \cdot \boldsymbol{p} p_{x} \\
0= & p_{x}+\lambda j_{y}^{\star}-\lambda \boldsymbol{j}^{\star} \cdot \boldsymbol{p} p_{y} \\
0= & \lambda j_{z}^{\star}-\lambda \boldsymbol{j}^{\star} \cdot \boldsymbol{p} p_{z} .
\end{array}\right.
$$

By making use of the third equation of system (108), one can easily check that the system admits exactly two equilibrium orientations, $\boldsymbol{p}_{\mathrm{eq}}^{ \pm}=\left(p_{x}^{ \pm}, p_{y}^{ \pm}, p_{z}^{ \pm}\right)$satisfying

$$
p_{z}^{ \pm}= \pm \sqrt{\frac{1-\lambda^{2}+\sqrt{\left(1-\lambda^{2}\right)^{2}+4 \lambda_{0}^{2} \kappa^{2}}}{2}}
$$

and

$$
p_{x}^{ \pm}=\frac{-\lambda_{0} y p_{z}^{2}-\lambda_{0}^{2} \kappa x p_{z}^{ \pm}}{p_{z}^{2}+\lambda_{0}^{2} \kappa^{2}}, \quad p_{y}^{ \pm}=\frac{\lambda_{0} x p_{z}^{2}-\lambda_{0}^{2} \kappa y p_{z}^{ \pm}}{p_{z}^{2}+\lambda_{0}^{2} \kappa^{2}}
$$


The ' + ' solution is stable and the '-' solution is unstable. Note that for $(x, y)=0$ we have $\boldsymbol{j}^{\star}=(0,0,1)$. In this case, it is shown (see Section V.B.1) that $\boldsymbol{p}$ tends to $\boldsymbol{k}$ (opposite to gravity) as $t$ tends to infinity.

As above, it is possible to analytically show that swimmers accumulate exponentially in time at the $z-$ axis. By making use of the position equation, one deduces that the time evolution of the radial position $\left(\rho=\sqrt{x^{2}+y^{2}}\right)$ and the $z-$ component are described by

$$
\frac{d \rho}{d t}=-v_{s} \kappa \lambda_{0}^{2} \rho \frac{p_{z}^{+}}{p_{z}^{2}+\lambda_{0}^{2} \kappa^{2}}, \quad \frac{d z}{d t}=v_{s} p_{z}^{+}
$$

We see therefore that $\rho$ tends to 0 as $t$ tends to infinity and, in addition, the equations in (111) behave as, for large $t$,

$$
\frac{d \rho}{d t}=-v_{s} \kappa \lambda_{0}^{2} \frac{\rho}{1+\lambda_{0}^{2} \kappa^{2}}, \quad \frac{d z}{d t}=v_{s} .
$$

Therefore, for large $t, z(t)=v_{s} t$, and the radial position (the distance from the rotation axis) asymptotically tends to 0 for $t \rightarrow \infty$ as

$$
\rho(t)=\text { const. } e^{-\frac{v_{s} \kappa \lambda_{0}^{2}}{1+\lambda_{0}^{2} \kappa^{2}} t} .
$$

This result deviates from that obtained in [29]. In fact, in [29], it was argued that the stable equilibrium is simply $-\boldsymbol{A} / \| \boldsymbol{A} \mid$, which is valid only if the vorticity is neglected (see Eqs. (100) and (101)). This leads to the asymptotic solution for radial distance (105), with time constant $v_{s} / \kappa$ instead of $v_{s} \kappa \lambda_{0}^{2} /\left(1+\lambda_{0}^{2} \kappa^{2}\right)$, given by our exact asymptotic solution (113)

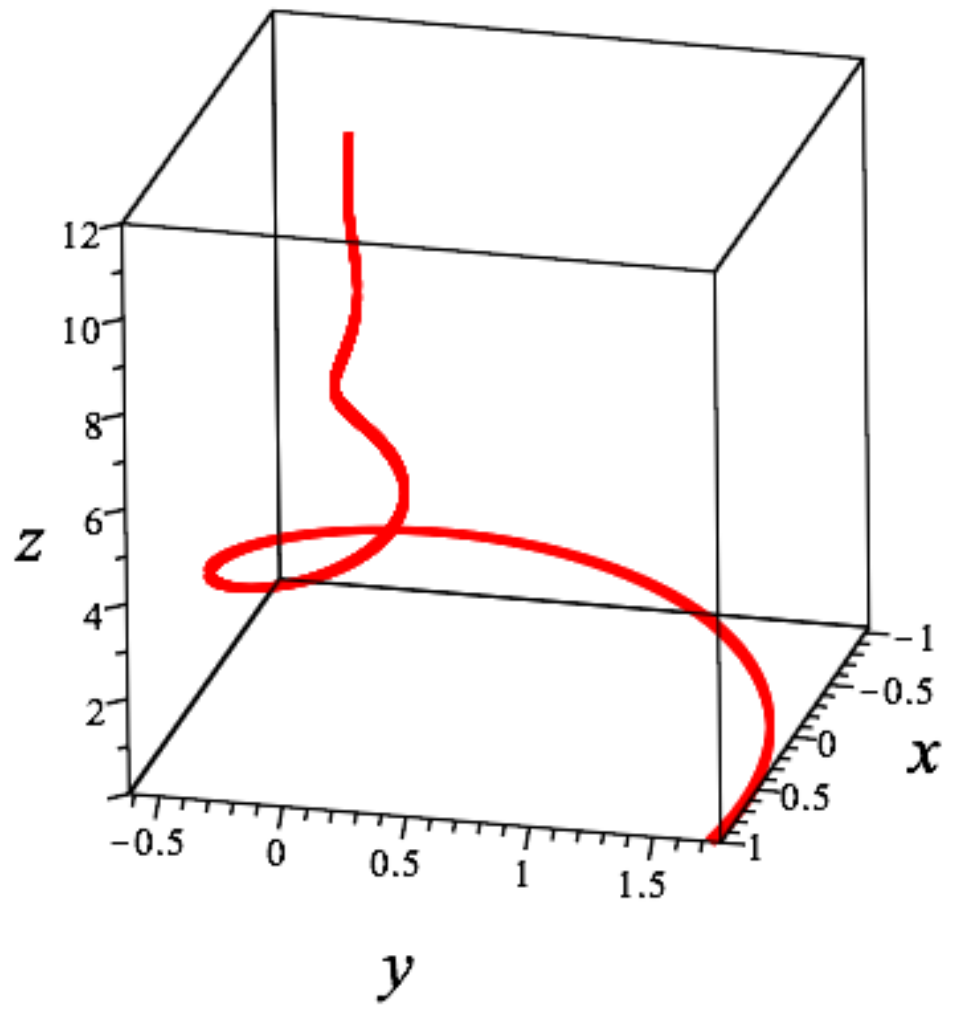

FIG. 11. An example of trajectories, for large $t$, of a gyrotactic swimmer in a fluid acceleration. Parameters are $\dot{\gamma}=1, \kappa=1, v_{s}=1, a_{0}=2$ and $\theta_{0}=\pi / 3$. The particle reaches the $z-$ axis.

Making use of the results of this section we can write

$$
\frac{1}{\mathbf{x}_{\mathbf{c}}} \frac{d \mathbf{x}_{\mathbf{c}}}{d t}=\frac{1}{\rho} \frac{d \rho}{d t}+i\left[\dot{\gamma}+v_{s} \lambda_{0} \frac{p_{z}^{2}}{p_{z}^{2}+\lambda_{0}^{2} \kappa^{2}}\right],
$$


which leads to

$$
\mathbf{x}_{c}(t)=\mathbf{x}_{c}(0) \frac{\rho(t)}{\rho(0)} e^{i\left(\dot{\gamma} t+v_{s} \lambda_{0} \int_{0}^{t} \frac{p_{z}^{2}}{p_{z}^{2}+\lambda_{0}^{2} \kappa^{2}} d s\right)} .
$$

The above result shows that the swimmer oscillates an infinite number of times around the rotation axis and reaches this axis for $t \rightarrow \infty$.

An approximate particle trajectory, for large $t$, can be found by using again Eq. (114), or Eq. (115). It is found that (see Figure 11)

$$
\left\{\begin{array}{l}
x(t) \sim a_{0} \rho(t) \cos \left(\left(\dot{\gamma}+\frac{v_{s} \lambda_{0}}{1+\lambda_{0}^{2} \kappa^{2}}\right) t+\theta_{0}\right), \\
y(t) \sim a_{0} \rho(t) \sin \left(\left(\dot{\gamma}+\frac{v_{s} \lambda_{0}}{1+\lambda_{0}^{2} \kappa^{2}}\right) t+\theta_{0}\right), \\
z(t) \sim v_{s} t
\end{array}\right.
$$

where $\rho$ is given by (113) and $a_{0}$ and $\theta_{0}$ are real parameters.

In summary, in the presence of gravity alone we have a pseudo-attractor (final trajectory depends on initial condition, see Eq.(88)). When only fluid acceleration is present, the trajectory tends towards a limit cycle at finite time (see Eqs. (97)-98)). When both gravity and fluid acceleration are present, the trajectory focuses towards the cylinder axis at long time (see Eq.(113)).

\section{Stagnation point flow}

This is our final example corresponding to a swimmer placed in a two-dimensional stagnation point flow. The velocity profile of the flow is known to be given by

$$
\boldsymbol{u}_{0}=(\delta x,-\delta y, 0)
$$

where $\delta$ is a positive constant, or by the stream function $\Psi=\delta x y$.

The particularity of the above profile is that the flow has zero vorticity. Thus, the equation of orientation for $\boldsymbol{p}$ (Eq. (5)) reads (see Eq. (100))

$$
\dot{p}=\frac{1}{2} \lambda\left(j^{\star}-j^{\star} \cdot p p\right) .
$$

The stagnation flow makes the mathematical study relatively simple. Eq. (118) has two equilibrium orientations $\pm \boldsymbol{j}^{\star}$, and that only the ' + ' equilibrium orientation is stable; $\boldsymbol{p}$ tends to $\boldsymbol{j}^{\star}$ as $\mathrm{t}$ approaches infinity.

It is a simple matter to show that the trajectory is given by, with $\boldsymbol{j}^{\star}=\left(\boldsymbol{j}_{1}^{\star}, \boldsymbol{j}_{2}^{\star}, \boldsymbol{j}_{3}^{\star}\right)$,

$$
\begin{gathered}
x(t)=\left(x(0)+\frac{v_{s}}{\delta} \boldsymbol{j}_{1}^{\star}\right) e^{\delta t}-\frac{v_{s}}{\delta} \boldsymbol{j}_{1}^{\star}, \quad y(t)=\left(y(0)-\frac{v_{s}}{\delta} \boldsymbol{j}_{2}^{\star}\right) e^{-\delta t}+\frac{v_{s}}{\delta} \boldsymbol{j}_{2}^{\star}, \\
z(t)=z(0)+v_{s} \boldsymbol{j}_{3}^{\star} t .
\end{gathered}
$$

By making use of (119) one deduces

$$
\left(x+\frac{v_{s}}{\delta} \boldsymbol{j}_{1}^{\star}\right)\left(y-\frac{v_{s}}{\delta} \boldsymbol{j}_{2}^{\star}\right)=\left(x(0)+\frac{v_{s}}{\delta} \boldsymbol{j}_{1}^{\star}\right)\left(y(0)-\frac{v_{s}}{\delta} \boldsymbol{j}_{2}^{\star}\right),
$$

showing that the $x-y$ plane projections follow the streamlines; the particle trajectory is a hyperbola (see Figure 12.)

For the case where parameter $\lambda$ is negative, we start from Eq. (100) with $-\boldsymbol{j}^{\star}$ instead of $\boldsymbol{j}^{\star}$. Repeating the above analysis we deduce that swimmer direction $\boldsymbol{p}$ tends to $-\boldsymbol{j}^{\star}$, as $t$ tends to infinity, and the swimmer trajectory satisfies (119), (120) with $-\boldsymbol{j}_{i}^{\star}, i=1,2,3$, instead of $\boldsymbol{j}_{i}^{\star}$.

\section{SUMMARY OF THE MAIN RESULTS}

We provide in Table I below a summary of different trajectories obtained for different flows and external forces. 


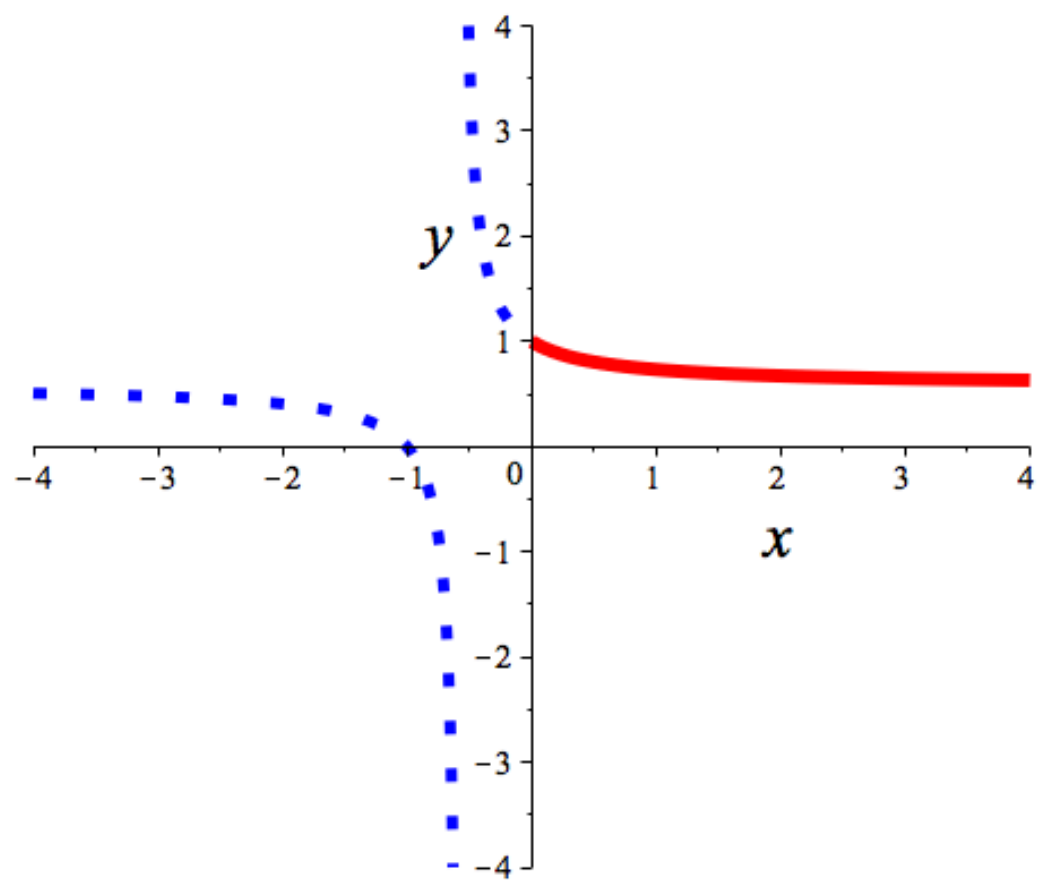

FIG. 12. Projection of a particle trajectory in the $x-y$ plane according to Eq. (121). Parameters are $\delta=1, v_{s}=1$ and $\boldsymbol{j}_{i}^{\star}=1 / \sqrt{3}, i=1,2,3$. The initial condition is $x(0)=0$ and $y(0)=1$, for solid red curve which is a part of the full hyperbolic trajectory (121); the complementary trajectory is shown as a blue dotted line.

\section{CONCLUSION}

One of the major investigations of the paper is to analytically solve a family of equations of the type

$$
\dot{\mathbf{p}}=\frac{1}{2}\left[\mathbf{\Omega} \cdot \boldsymbol{p}+\lambda\left(\boldsymbol{j}^{\star}-\boldsymbol{j}^{\star} \cdot \boldsymbol{p} \boldsymbol{p}\right)\right]
$$

in which $\lambda$ is a real (physical) parameter, $\boldsymbol{\Omega}$ is an arbitrary vorticity tensor that may contains all components of the fluid vorticity $\omega$ and $j^{\star}$ is an arbitrary (unit) vector. Equation (122) describes the time evolution of the orientation of a spherical particle in an arbitrary flow and subject to an external force $j^{\star}$.

We have shown that a solution to the above problem can be given explicitly. We have studied a (general) model of spherical gravitactic or magnetotactic swimmer and have analyzed its orientation and trajectories in general linear flows. Firstly, we have shown how to solve easily the problem in the spirit of [25] and then we have obtained exact analytical solutions for the orientation and the trajectory of the particle. Depending on parameter $\lambda$, it is found that the particle can perform run or tumble dynamics. In addition, we have derived analytically several swimming trajectories. The particle orientations and trajectories can be quantitatively and qualitatively controlled by an external field (e.g. gyrotactic or magnetic field). A natural extension is to study this problem for other flows (e.g; Poiseuille flow) and for non spherical particles. We hope to investigate this matter in a future work.

Acknowledgments. The present work was funded by Project T-MEBIO (AAP A2U 2019), CNES (Centre National d'Etudes Spatiales) and by the French-German University Programme Living Fluids (grant CFDA-Q1-14).

\section{Appendix A: Adoption of Betherton solution to our problem}

Without any algebraic transformation, it seems difficult to apply or follow the Bretherton approach directly due to the structure of Eq. (5), which is clearly different from (8). The main idea is to transform this equation into an equation for which the analysis is particularly simple in the spirit of [25].

To solve Eq. (5) we first use the Ansatz

$$
\boldsymbol{p}=\boldsymbol{p}_{0}+\alpha(t) \boldsymbol{q}
$$




\begin{tabular}{|c|c|c|}
\hline Type of flow and force & Run regime & Tumble regime \\
\hline $\begin{array}{c}\text { Linear shear flow } \\
\mathbf{u}_{0}=(\dot{\gamma} y, 0,0), \boldsymbol{\omega}=-\dot{\gamma} \boldsymbol{k}\end{array}$ & Parabolic trajectory & Inclined Helix \\
\hline \multicolumn{3}{|l|}{$\begin{array}{c}\text { 2d Linear flow } \\
\mathbf{u}_{0}=\left(\dot{\gamma}_{1} y, \dot{\gamma}_{2} x, 0\right), \boldsymbol{\omega}=\left(\dot{\gamma}_{2}-\dot{\gamma}_{1}\right) \boldsymbol{k}\end{array}$} \\
\hline \multicolumn{3}{|l|}{$\begin{array}{l}\text { Vorticity perpendicular to force } \\
\qquad \boldsymbol{g}=g \boldsymbol{j}\end{array}$} \\
\hline$\dot{\gamma}_{1} \dot{\gamma}_{2}<0$ & $\begin{array}{l}\text { Hyperboloic trajectory in } x-y \\
\quad z=\text { constant }\end{array}$ & Spherical Helix \\
\hline$\dot{\gamma}_{1} \dot{\gamma}_{2}>0$ & $\begin{array}{l}\text { Parabolic trajectory in } x-y \\
\quad z=\text { constant }\end{array}$ & No analytical solution \\
\hline \multicolumn{3}{|l|}{$\begin{array}{l}\text { Vorticity parallel to force } \\
\qquad g=-g k\end{array}$} \\
\hline$\dot{\gamma}_{1} \dot{\gamma}_{2}>0$ & Hyperbolic trajectory in $x-y, z \sim t$ & No tumbling \\
\hline$\dot{\gamma}_{1} \dot{\gamma}_{2}<0$ & $\begin{array}{l}\text { Elliptical trajectory in } x-y, z \sim t \\
\text { 3D trajectory is elliptical helix }\end{array}$ & No tumbling \\
\hline \multicolumn{3}{|l|}{$\begin{array}{c}\text { Rigid body rotation } \\
\mathbf{u}_{0}=-\dot{\gamma}(y,-x, 0), \boldsymbol{\omega}=2 \dot{\gamma} \boldsymbol{k} \\
\text { External force } \boldsymbol{A}=\boldsymbol{g}-\boldsymbol{a} \\
\boldsymbol{a}=\left(-\dot{\gamma}^{2} x,-\dot{\gamma}^{2} y, 0\right)\end{array}$} \\
\hline$A=g=-g k$ & $\begin{array}{c}\text { Run along } k \text { with helical trajectory } \\
\text { with radius depending on initial condition }\end{array}$ & No tumbling \\
\hline$A=-a$ & $\begin{array}{l}\text { particle reaches cylinder } \\
\mathscr{A}=\left\{\dot{\gamma}^{2}\left(x^{2}+y^{2}\right)=4 v_{0}^{2}\right\} \\
\quad \text { at finite time } t^{*}\end{array}$ & No tumbling \\
\hline$A=g-a$ & $\begin{array}{c}\text { Particle reaches cylinder axis } \\
\text { with radius behaving as } \rho(t)=\text { const. } e^{-\frac{v_{s} \kappa \lambda_{0}^{2}}{1+\lambda_{0}^{2} \kappa^{2}} t}\end{array}$ & No tumbling \\
\hline $\begin{array}{c}\text { Stagnation flow } \\
\boldsymbol{u}_{0}=(\delta x,-\delta y, 0)\end{array}$ & $\begin{array}{c}\text { Run along } j^{*}, \text { an arbitrary force direction } \\
\text { with hyperbolic trajectory in } x-y, z=\text { constant }\end{array}$ & No tumbling \\
\hline
\end{tabular}

TABLE I. Summary of different trajectories obtained analytically

where $\boldsymbol{p}_{0}$ is a steady unknown vector and $\alpha$ and $\boldsymbol{q}$ are unsteady unknown quantities. Our idea is to split the resulting equation into three equations. The first equation is that satisfied by $\boldsymbol{p}_{0}$ :

$$
\Omega \cdot p_{0}+\lambda j^{\star}-\lambda j^{\star} \cdot p_{0} p_{0}=0,
$$

which is nothing but the stationary version of (5).

The second equation is that obeyed by $\boldsymbol{q}$ :

$$
\dot{q}=\frac{1}{2}\left[\Omega \boldsymbol{q}-\lambda \boldsymbol{j}^{\star} \cdot \boldsymbol{q} \boldsymbol{p}_{0}\right]
$$

This equation is linear and its solution is given by

$$
\boldsymbol{q}(t)=\exp \left(\frac{1}{2} \tilde{\Omega}_{0} t\right) \boldsymbol{q}(0)
$$

where matrix $\tilde{\Omega}_{0}$ is defined by

$$
\tilde{\mathbf{\Omega}}_{0}=\boldsymbol{\Omega}-\lambda \boldsymbol{p}_{0} \boldsymbol{j}^{\star \mathrm{T}}
$$

or, equivalently,

$$
\tilde{\mathbf{\Omega}}_{0} \cdot \boldsymbol{q}=\mathbf{\Omega} . \boldsymbol{q}-\lambda \boldsymbol{j}^{\star} \cdot \boldsymbol{q} \boldsymbol{p}_{0} .
$$

Finally, the equation of $\alpha$ is found to be

$$
\frac{d \alpha}{d t}=-\frac{\lambda}{2}\left[\alpha \boldsymbol{j}^{\star} \cdot \boldsymbol{p}_{0}+\alpha^{2} \boldsymbol{j}^{\star} \cdot \boldsymbol{q}\right] .
$$


The general solution to the above ordinary differential equation, which is of Bernoulli-type, can be expressed as

$$
\alpha^{-1}(t)=\alpha^{-1}(0) e^{\frac{\lambda}{2} j^{\star} \cdot p_{0} t}+\frac{\lambda}{2} \int_{0}^{t} e^{\frac{\lambda}{2} j^{\star} \cdot p_{0}(t-s)} j^{\star} \cdot q(s) d s,
$$

once the expression of $\boldsymbol{p}_{0}$ (to be determined below), and the expression of $\boldsymbol{q}$ are known (Eq. (A4)). Equation (A8) is simply derived by making the change of variable $\alpha \rightarrow \alpha^{-1}$. Therefore, any solution to Eq. (5) can be written as

$$
\boldsymbol{p}(t)=\boldsymbol{p}_{0}+\frac{\exp \left(\frac{1}{2} \tilde{\boldsymbol{\Omega}}_{0} t\right) \boldsymbol{q}(0)}{\alpha^{-1}(0) e^{\frac{\lambda}{2} \boldsymbol{j}^{\star} \cdot \boldsymbol{p}_{0} t}+\frac{\lambda}{2} \int_{0}^{t} e^{\frac{\lambda}{2} \boldsymbol{j}^{\star} \cdot \boldsymbol{p}_{0}(t-s)} \boldsymbol{j}^{\star} \cdot \exp \left(\frac{1}{2} \tilde{\boldsymbol{\Omega}}_{0} s\right) \boldsymbol{q}(0) d s} .
$$

In expression (A9), $\alpha(0)$ and $\boldsymbol{q}(0)$ (initial conditions) have to be chosen such that $|\boldsymbol{p}|=1$. Here, we shall see that exact solutions for various cases can be framed in terms of Eq. (A9). The general expression of $\boldsymbol{p}$ is interpreted as a linear superposition of independent solutions to Eq. (A3).

For the particular and interesting case in which $\boldsymbol{j}^{\star}$ and $\boldsymbol{p}_{0}$ are perpendicular (this orthogonality will naturally emerge, as discussed below), Eq. (A9) reduces to

$$
\boldsymbol{p}(t)=\boldsymbol{p}_{0}+\frac{\exp \left(\frac{1}{2} \tilde{\boldsymbol{\Omega}}_{0} t\right) \boldsymbol{q}(0)}{\alpha^{-1}(0)+\frac{\lambda}{2} \int_{0}^{t} \boldsymbol{j}^{\star} \cdot \exp \left(\frac{1}{2} \tilde{\boldsymbol{\Omega}}_{0} s\right) \boldsymbol{q}(0) d s}
$$

We only need now to determine $\boldsymbol{p}_{0}$ given by Eq. (A2). A priori, a solution $\boldsymbol{p}_{0}$ to (A2) satisfies

$$
\boldsymbol{j}^{\star} \cdot \boldsymbol{p}_{0}\left(1-\left|\boldsymbol{p}_{0}\right|^{2}\right)=0
$$

indicating that any solution to (A2) has to satisfy either the unit norm condition, $\left|\boldsymbol{p}_{0}\right|=1$, or the orthogonality condition with $\boldsymbol{j}^{\star}$

$$
j^{\star} \cdot p_{0}=0
$$

If this solution is selected, it is easily seen from Eq. (A2) that this implies

$$
\omega \cdot j^{\star}=0
$$

If this orthogonality between external field and vorticity is not fulfilled (see later), one has thus to consider the other solution of Eq. (A11) $\left|\boldsymbol{p}_{0}\right|=1$, which is not sufficient to determine a full solution of $\boldsymbol{p}_{0}$. We have thus to go back to (A2) and to analyze it explicitly. An example is treated in Section V C.

In the frequent cases where the orthogonality condition (A12) is satisfied, we obtain (see Appendix B) a ( $\xi$, $\varepsilon$ )-family of solutions satisfying this equation, which can be decomposed as

$$
p_{0}=\xi \omega+\lambda \Omega^{\star}(\varepsilon) \cdot j^{\star}
$$

where $\xi \in \mathbb{R}$ and $\varepsilon=\left(\varepsilon_{1}, \varepsilon_{2}, \varepsilon_{3}\right) \in \mathbb{R}^{3}$, such that $\varepsilon_{1}+\varepsilon_{2}+\varepsilon_{3}=1$, are parameters. Matrix $\Omega^{\star}(\varepsilon)$ is the vorticity or rotation matrix associated to (vorticity) vector $\omega^{\star}(\varepsilon)=\left(\varepsilon_{1} / \omega_{1}, \varepsilon_{2} / \omega_{2}, \varepsilon_{3} / \omega_{3}\right)$, with the convention $\varepsilon_{m}=0$ and $\varepsilon_{m} / \omega_{m}=0$ if $\omega_{m}=0$. Equation (A14) can also be written as

$$
p_{0}=\xi \omega+\lambda \omega^{\star}(\varepsilon) \times j^{\star}
$$

A more practical choice of $\boldsymbol{\varepsilon}=\left(\varepsilon_{1}, \varepsilon_{2}, \varepsilon_{3}\right)$ would be to take $\varepsilon_{i}=\omega_{i}^{2} / \omega^{2}, i=1,2,3$. In this case (A15) simply reads

$$
p_{0}=\xi \omega+\frac{\lambda}{\omega^{2}} \omega \times j^{\star}
$$

The solution (A10) with $\boldsymbol{p}_{0}$ given by (A15), or (A16), will constitute our basic solution of the model equation (5), which will be used now in order to determine the explicit expressions of $\boldsymbol{p}(t)$ and the particle trajectory. Before embarking on our explicit calculations, we stress that expression (A10) contains parameter $\xi$ (in view of (A15)), which plays the same role as the Jeffery orbit constant (it can be checked in our explicit calculations below that it can be absorbed into the Jeffery constant). 


\section{Appendix B: Solution of $p_{0}$}

Looking for a stationary (pseudoequilibrium) solution to (5), or to Eq. (A2), satisfying (A12), one sees that this solution is also a solution to the following homogeneous linear system

$$
\Omega \cdot p_{0}=-\lambda j^{\star}
$$

which appears to be amenable to a simple analytical treatment, despite the fact that matrix $\Omega$ is singular. Most importantly, since $\boldsymbol{p} . \Omega . \boldsymbol{p}=0$, for any vector $\boldsymbol{p}$, any solution to (B1), when it exists, is a pseudo-equilibrium orientation in the sense that Eq. (5) is automatically satisfied.

Thus, it is more convenient to examine Eq. (B1). Since the matrix we are dealing with is singular, as mentioned before, it is known that Eq. (B1) has no solution or infinitely many solutions. Moreover, any (possible) solution can be written as

$$
\boldsymbol{p}_{0}=\boldsymbol{p}_{0}^{h}+\boldsymbol{p}_{0}^{p}
$$

where $\boldsymbol{p}_{0}^{h}$ is a solution of the homogenous system

$$
\Omega \cdot p_{0}^{h}=0
$$

and $\boldsymbol{p}_{0}^{p}$ is a particular solution to (B1). Clearly, Eq. (B3) is automatically satisfied if $\boldsymbol{p}_{0}^{h}=\omega$ and the orthogonality condition (A12) is also satisfied if $\boldsymbol{p}_{0}^{p}$ is written as

$$
p_{0}^{p}=\omega^{\prime} \times j^{\star}
$$

for some vector $\omega^{\prime}$. However, it is not obvious if Eq. (B1) admits a (particular) solution and if this solution can be represented as Eq. (B4).

In fact, it is readily seen, on the one hand, that Eq. (B1) has (multiple) solutions only if the fluid vorticity and the external field are perpendicular. On the other hand, under the orthogonality condition, we obtain a $(\xi, \varepsilon)-$ family of pseudo-equilibrium solutions that can be decomposed as

$$
p_{0}=\xi \omega+\lambda \Omega^{\star}(\varepsilon) \cdot j^{\star}
$$

where $\xi \in \mathbb{R}$ and $\varepsilon=\left(\varepsilon_{1}, \varepsilon_{2}, \varepsilon_{3}\right) \in \mathbb{R}^{3}$, such that $\varepsilon_{1}+\varepsilon_{2}+\varepsilon_{3}=1$, are parameters. Matrix $\Omega^{\star}(\varepsilon)$ is the vorticity or rotation matrix associated to (vorticity) vector $\omega^{\star}(\varepsilon)=\left(\varepsilon_{1} / \omega_{1}, \varepsilon_{2} / \omega_{2}, \varepsilon_{3} / \omega_{3}\right)$, with the convention $\varepsilon_{m}=0$ and $\varepsilon_{m} / \omega_{m}=0$ if $\omega_{m}=0$. This is our central result for Eq. (B1). Eq. (A14) can also be written as

$$
p_{0}=\xi \omega+\lambda \omega^{\star}(\varepsilon) \times j^{\star}
$$

Let us observe that $\omega^{\star}(\varepsilon)$ describes an infinite number of (vorticity) vectors satisfying

$$
\omega . \omega^{\star}(\varepsilon)=1 \text {. }
$$

The corresponding (vorticity) matrix is given by

$$
\Omega^{\star}(\varepsilon)=\left(\begin{array}{ccc}
0 & -\frac{\varepsilon_{3}}{\omega_{3}} & \frac{\varepsilon_{2}}{\omega_{2}} \\
\frac{\varepsilon_{3}}{\omega_{3}} & 0 & -\frac{\varepsilon_{1}}{\omega_{1}} \\
-\frac{\varepsilon_{2}}{\omega_{2}} & \frac{\varepsilon_{1}}{\omega_{1}} & 0
\end{array}\right),
$$

which can be written as

$$
\Omega^{\star}(\varepsilon)=\frac{\varepsilon_{1}}{\omega_{1}} \Omega_{1}+\frac{\varepsilon_{2}}{\omega_{2}} \Omega_{2}+\frac{\varepsilon_{3}}{\omega_{3}} \Omega_{3},
$$

where $\Omega_{i}^{\star}, i=1,2,3$ is the usual basis of rotation matrices in $\mathbb{R}^{3}$.

We would like to stress that any (pseudo-equilibrium) $\boldsymbol{p}_{0}$ given by (A14) satisfies $\boldsymbol{p}_{0} \cdot \boldsymbol{j}^{\star}=\boldsymbol{\xi} \omega$. $\boldsymbol{j}^{\star}$. This observation, supplemented with the above analysis, may provide a natural explanation of the orthogonality condition between the external field and the fluid vorticity to find exact analytical solutions. In fact, the orthogonality condition is necessary and sufficient to solve the 
pseudo-equilibrium equation under the additional assumption $\boldsymbol{p}_{0} \cdot \boldsymbol{j}^{\star}=0$, which, as mentioned before, simplifies the construction of the exact general expression (A9).

A more practical choice of $\boldsymbol{\varepsilon}=\left(\varepsilon_{1}, \varepsilon_{2}, \varepsilon_{3}\right)$ would be to take $\varepsilon_{i}=\omega_{i} / \omega^{2}, i=1,2,3$ (recall that $\left.\omega=\|\boldsymbol{\omega}\|\right)$. In this case $(\mathrm{A} 15)$ simply reads

$$
p_{0}=\xi \omega+\frac{\lambda}{\omega^{2}} \omega \times j^{\star} .
$$

Let us note that since a pseudo-equilibrium orientation given by (B10) satisfies

$$
\left\|p_{0}\right\|^{2}=\xi^{2} \omega^{2}+\frac{\lambda^{2}}{\omega^{2}},
$$

we get an equilibrium orientation for

$$
\xi^{2} \omega^{2}=1-\frac{\lambda^{2}}{\omega^{2}}
$$

provided that $1>\frac{\lambda^{2}}{\omega^{2}}$.

Once a pseudo-equilibrium is known (see (A14)), our approach can also be used to derive the exact expression of the equilibrium orientations of Eq. (5) and to easily study their stability without calculating the exact general expression of $\boldsymbol{p}$ (see (A9)). This is a further evidence of our approach. According to Ansatz (A1), orientation $\boldsymbol{p}$ can be written as

$$
\boldsymbol{p}=\boldsymbol{p}_{0}+\tilde{\mathbf{p}}
$$

where $\tilde{\mathbf{p}}$ satisfies

$$
\dot{\tilde{\mathbf{p}}}=\frac{1}{2}\left[\tilde{\Omega}_{0} \cdot \tilde{\mathbf{p}}-\lambda\left(\boldsymbol{j}^{\star} \cdot \tilde{\mathbf{p}}\right) \tilde{\mathbf{p}}\right]
$$

where matrix $\tilde{\Omega}_{0}$ is given in (A5) or (A6). This suggests that an equilibrium orientation may be written as

$$
\boldsymbol{p}_{\mathrm{eq}}=\boldsymbol{p}_{0}+\tilde{\boldsymbol{p}}_{\text {eg }}
$$

where $\tilde{\boldsymbol{p}}_{e g}$ is an equilibrium solution to (B14);

$$
\tilde{\Omega}_{0} \cdot \tilde{\boldsymbol{p}}_{e g}=\lambda \boldsymbol{j}^{\star} \cdot \tilde{\boldsymbol{p}}_{e g} \tilde{\boldsymbol{p}}_{e g}
$$

Even if the above nonlinear equation seems to be difficult to solve, in general, it suffices to note that Eq. (B16) indicates that $\tilde{\boldsymbol{p}}_{e g}$ and $\lambda \boldsymbol{j}^{\star} . \tilde{\boldsymbol{p}}_{e g}$ are an eigenvector and an eigenvalue, respectively, of matrix $\tilde{\Omega}_{0}$. Therefore, if $\tilde{\boldsymbol{p}}_{0}$ is an eigenvector of matrix $\tilde{\Omega}_{0}$ associated to an eigenvalue $\chi \neq 0$, a solution to Eq. (B16) can be written as

$$
\tilde{\boldsymbol{p}}_{e g}=\frac{\chi}{\lambda \boldsymbol{j}^{\star} \cdot \tilde{\boldsymbol{p}}_{0}} \tilde{\boldsymbol{p}}_{0}
$$

if $\boldsymbol{j}^{\star} \cdot \tilde{\boldsymbol{p}}_{0} \neq 0$, and then

$$
\boldsymbol{p}_{\text {eq }}=\boldsymbol{p}_{0}+\frac{\chi}{\lambda \boldsymbol{j}^{\star} \cdot \tilde{\boldsymbol{p}}_{0}} \tilde{\boldsymbol{p}}_{0} .
$$

If $\boldsymbol{j}^{\star} \cdot \tilde{\boldsymbol{p}}_{0}=0$, we use (A6) to deduce firstly that $\tilde{\boldsymbol{p}}_{0}$ is also an eigenvector of matrix $\Omega$ and then $\tilde{\boldsymbol{p}}_{0}=0$, from which we get

$$
\boldsymbol{p}_{\mathrm{eq}}=\boldsymbol{p}_{0}
$$

provided that $\left|\boldsymbol{p}_{0}\right|=1$, otherwise we obtain a contradiction and then $\boldsymbol{j}^{\star} \cdot \tilde{\boldsymbol{p}}_{0} \neq 0$.

To check the stability of the stationary solutions of Eq. (B14) and then the stability of $\boldsymbol{p}_{\text {eq }}$, we recall that the solution of Eq. (B14) with initial condition $\tilde{\boldsymbol{p}}(0)$ has been shown to be

$$
\tilde{\boldsymbol{p}}(t)=\frac{\exp \left(\frac{1}{2} \tilde{\Omega}_{0} t\right) \tilde{\boldsymbol{p}}(0)}{1+\frac{\lambda}{2} \int_{0}^{t} j^{\star} \cdot \exp \left(\frac{1}{2} \tilde{\Omega}_{0} s\right) \tilde{\boldsymbol{p}}(0) d s} .
$$


In the following we denote by $\chi_{d}$ the real eigenvalue of matrix $\tilde{\Omega}_{0}$ (if any) which strictly dominates (the real part of) other eigenvalues. Therefore, it is easy to see that if $\chi_{d}>0$, the corresponding eigenvector, $\tilde{\boldsymbol{p}}_{0}^{d}$, is the unique stable stationary solution of Eq. (B14). Therefore, orientation $\boldsymbol{p}$ tends to $\boldsymbol{p}_{\mathrm{eq}}$, as $t$ approaches infinity, where

$$
\boldsymbol{p}_{\mathrm{eq}}=\boldsymbol{p}_{0}+\frac{\chi_{d}}{\lambda \boldsymbol{j}^{\star} \cdot \tilde{\boldsymbol{p}}_{0}^{d}} \tilde{\boldsymbol{p}}_{0}^{d}
$$

Let us note that since $\boldsymbol{p}_{0}$ and $\boldsymbol{j}^{\star}$ (or $\boldsymbol{j}^{\star}$ and $\boldsymbol{\omega}$ ) are perpendicular the stability of $\boldsymbol{p}_{\text {eq }}$ requires that $\lambda \boldsymbol{j}^{\star} \cdot \boldsymbol{p}_{\text {eq }}>0$. Similar conclusion is derived in [7] by a linearized stability analysis involving a small perturbation from $\boldsymbol{p}_{\mathrm{eq}}$ and perpendicular to it. In fact, it is not difficult to demonstrate that the condition $\lambda \boldsymbol{j}^{\star} \cdot \boldsymbol{p}_{\mathrm{eq}}>0$ is necessary and sufficient for the (strict) stability of $\boldsymbol{p}_{\mathrm{eq}}$ given by (B21). Let us note that if matrix $\tilde{\Omega}_{0}$ has exactly one real eigenvalue $\chi_{r}$ such that $\chi_{r} \leq 0$, then $\boldsymbol{p}$ exhibits a tumble regime.

By way of illustration without excessive calculation, we firstly consider two cases. Each of these cases may in turn be carried out in a variety of practical situations. Recall here that we are concerned with exact solutions to Eq. (5) in which $\boldsymbol{j}^{\star}$ is perpendicular to vorticity $\boldsymbol{\omega}$ in most cases.

1. $\omega_{1}=\omega_{2}=0$ and $\omega_{3} \neq 0$. The orthogonality condition is satisfied for $\boldsymbol{j}^{\star}=a \boldsymbol{i}+b \boldsymbol{j}$, where $a$ and $b$ are (arbitrary) real parameters such that $a^{2}+b^{2}=1$. For $\varepsilon$, there is only one choice; $\varepsilon_{1}=\varepsilon_{2}=0$ and $\varepsilon_{3}=1$. For $\boldsymbol{p}_{0}$, we may take $\xi=0$;

$$
p_{0}=\left(-\frac{\lambda}{\omega_{3}} b, \frac{\lambda}{\omega_{3}} a, 0\right)
$$

It follows from this that

$$
\tilde{\Omega}_{0}=\left(\begin{array}{ccc}
\frac{\lambda^{2} a b}{\omega_{3}} & -\omega_{3}+\frac{\lambda^{2} b^{2}}{\omega_{3}} & 0 \\
\omega_{3}-\frac{\lambda^{2} a^{2}}{\omega_{3}} & -\frac{\lambda^{2} a b}{\omega_{3}} & 0 \\
0 & 0 & 0
\end{array}\right)
$$

which has 0 and two nontrivial real or pure imaginary complex eigenvalues satisfying

$$
\chi^{2}=\lambda^{2}-\omega_{3}^{2} .
$$

Hence, we may conclude that we have two types of motions (run or tumble regime) depending on whether quantity $\lambda^{2}-\omega_{3}^{2}$ is positive or not.

Firstly, we consider the simple case in which (for instance) $\omega_{3}^{2}=\lambda^{2} a^{2}$. Hence, the spectrum of matrix $\tilde{\Omega}_{0}$ is given by $\sigma\left(\tilde{\Omega}_{0}\right)=\left\{0, \pm \lambda^{2} a b / \omega_{3}\right\}$. Clearly, we have $\chi_{d}=\lambda^{2} a b / \omega_{3}$ if $\lambda^{2} a b / \omega_{3}>0$. The corresponding eigenvector is shown to be $\tilde{\boldsymbol{p}}_{0}^{d}=(1,0,0)$ together with (B21) we deduce that

$$
\boldsymbol{p}_{\mathrm{eq}}=\frac{b}{|b|}(0,1,0) \text {. }
$$

In the similar way we get for the case $\chi_{d}=-\lambda^{2} a b / \omega_{3}>0$ the stable equilibrium orientation

$$
\boldsymbol{p}_{\mathrm{eq}}=\frac{b}{|b|}\left(2 a b, b^{2}-a^{2}, 0\right) \text {. }
$$

Next, we consider the case where $\lambda^{2}-\omega_{3}^{2}>0$ and assume that $\omega_{3}^{2} \neq \lambda^{2} a^{2}$ and $\omega_{3}^{2} \neq \lambda^{2} b^{2}$. The case $\omega_{3}^{2}=\lambda^{2} b^{2}$ which is similar to the case $\omega_{3}^{2}=\lambda^{2} a^{2}$ is left to the reader.

From (B24), we have $\chi_{d}=\sqrt{\lambda^{2}-\omega_{3}^{2}}$ and a simple calculation leads to the following eigenvector

$$
\tilde{\boldsymbol{p}}_{0}^{d}=\left(\frac{\lambda^{2} a b+\omega_{3} \sqrt{\lambda^{2}-\omega_{3}^{2}}}{\omega_{3}^{2}-\lambda^{2} a^{2}}, 1,0\right),
$$

or

$$
\tilde{\boldsymbol{p}}_{0}^{d}=\left(1, \frac{\lambda^{2} a b-\omega_{3} \sqrt{\lambda^{2}-\omega_{3}^{2}}}{\omega_{3}^{2}-\lambda^{2} b^{2}}, 0\right)
$$


which are collinear. The stable equilibrium orientation is then given by

$$
\boldsymbol{p}_{\mathrm{eq}}=\left(-\frac{\lambda}{\omega_{3}} b, \frac{\lambda}{\omega_{3}} a, 0\right)+\frac{\sqrt{\lambda^{2}-\omega_{3}^{2}}}{\lambda} \frac{1}{\boldsymbol{j}^{\star} \cdot \tilde{\boldsymbol{p}}_{0}^{d}} \tilde{\boldsymbol{p}}_{0}^{d},
$$

where $\tilde{\boldsymbol{p}}_{0}^{d}$ satisfies (B27) or (B28). For the special case in which $\boldsymbol{j}^{\star}=j(a=0, b=1)$ with $\lambda^{2}>\omega_{3}^{2}$, we deduce from (B27) and (B29)

$$
p_{\mathrm{eq}}=\left(-\frac{\omega_{3}}{\lambda}, \frac{1}{\lambda} \sqrt{\lambda^{2}-\omega_{3}^{2}}, 0\right)
$$

If we suppose that $\lambda^{2}<\omega_{3}^{2}$, we can see from (B13) and (B20) that any solution to (5) oscillates (tumble regime).

2. $\omega_{1}, \omega_{2} \neq 0, \omega_{3}=0$. In this case, we have different possibilities for $\varepsilon=\left(\varepsilon_{1}, 1-\varepsilon_{1}, 0\right)$, and for $\boldsymbol{j}^{\star}$. Here, setting $\boldsymbol{j}^{\star}=\boldsymbol{k}$ and making use of (A14), one sees that a pseudoequilibrium solution can be expressed as ( $\xi=0$ )

$$
p_{0}=\lambda\left(\frac{1-\varepsilon_{1}}{\omega_{2}},-\frac{\varepsilon_{1}}{\omega_{1}}, 0\right) .
$$

Matrix $\tilde{\Omega}_{0}$ reads

$$
\tilde{\Omega}_{0}=\left(\begin{array}{ccc}
0 & 0 & \omega_{2}+\frac{\lambda^{2}\left(\varepsilon_{1}-1\right)}{\omega_{2}} \\
0 & 0 & -\omega_{1}+\frac{\lambda^{2} \varepsilon_{1}}{\omega_{1}} \\
-\omega_{2} & \omega_{1} & 0
\end{array}\right),
$$

and has one null eigenvalue and two eigenvalues satisfying

$$
\chi^{2}=\lambda^{2}-\omega_{2}^{2}-\omega_{1}^{2},
$$

leading, as above, to run or tumble regime depending on whether quantity $\lambda^{2}-\omega_{1}^{2}-\omega_{2}^{2}$ is positive or negative.

For $\lambda^{2}>\omega_{1}^{2}+\omega_{2}^{2}$ (run regime), we have $\chi_{d}=\sqrt{\lambda^{2}-\omega_{1}^{2}-\omega_{2}^{2}}$ and the corresponding eigenvector can be written as

$$
\tilde{\boldsymbol{p}}_{0}^{d}=\left(\frac{\omega_{2}^{2}+\lambda^{2}\left(\varepsilon_{1}-1\right)}{\omega_{2} \chi_{d}}, \frac{-\omega_{1}^{2}+\lambda^{2} \varepsilon_{1}}{\omega_{1} \chi_{d}}, 1\right) .
$$

The analog of (B29) is

$$
\boldsymbol{p}_{\mathrm{eq}}=\lambda\left(\frac{1-\varepsilon_{1}}{\omega_{2}},-\frac{\varepsilon_{1}}{\omega_{1}}, 0\right)+\frac{\sqrt{\lambda^{2}-\omega_{1}^{2}-\omega_{2}^{2}}}{\lambda} \frac{1}{k \cdot \tilde{\boldsymbol{p}}_{0}^{d}} \tilde{\boldsymbol{p}}_{0}^{d}
$$

from which one obtains

$$
p_{\mathrm{eq}}=\left(\frac{\omega_{2}}{\lambda},-\frac{\omega_{1}}{\lambda}, \frac{\sqrt{\lambda^{2}-\omega_{1}^{2}-\omega_{2}^{2}}}{\lambda}\right) .
$$

It is important to notice that the particle motions or the eigenvalues of matrix $\tilde{\Omega}_{0}$ do not depend on the choice of parameter $\varepsilon_{1}$. We have seen, for example, that the choice $\boldsymbol{p}_{0}=\lambda / \omega_{2} \boldsymbol{i}\left(\varepsilon_{1}=0\right)$ is sufficient to derive an analytical expression for the orientation vector.

[1] E. M. Purcell, "Life at low reynolds number," American Journal of Physics 45, 3 (2012).

[2] E. Lauga and T. R. Powers, “The hydrodynamics of swimming microorganisms," Reports on Progress in Physics 72, 096601 (2009). 
[3] H. C. Berg, E. coli in Motion, Biological and Medical Physics, Biomedical Engineering (Springer, New York, 2004).

[4] L. Turner, W. S. Ryu, and H. C. Berg, "Real-time imaging of fluorescent flagellar filaments," Journal of Bacteriology 182, 2793-2801 (2000).

[5] A. Farutin, S. Rafai, D. K. Dysthe, A. Duperray, P. Peyla, and C. Misbah, "Amoeboid swimming: A generic self-propulsion of cells in fluids by means of membrane deformations," Phys. Rev. Lett. 111, 228102 (2013).

[6] H. Brenner, "Rheology of two-phase systems," Annual Review of Fluid Mechanics 2, 137-176 (1970).

[7] H. Brenner, "Rheology of a dilute suspension of dipolar spherical particles in an external field," Journal of Colloid and Interface Science 32, 141-158 (1970).

[8] W. F. Hall and S. N. Busenberg, "Viscosity of magnetic suspensions," The Journal of Chemical Physics 51, 137-144 (1969).

[9] J. Guckenheimer and P. Holmes, Nonlinear Oscillations, Dynamical Systems, and Bifurcations of Vector Fields, Applied Mathematical Sciences, Vol. 42 (Springer-Verlag New York, 1983).

[10] T. J. Pedley and J. O. Kessler, "The orientation of spheroidal microorganisms swimming in a flow field," Proceedings of the Royal Society of London. Series B. Biological Sciences 231, 47-70 (1987).

[11] J. O. Kessler, "Hydrodynamic focusing of motile algal cells," Nature 313, 218-220 (1985).

[12] A. Zottl and H. Stark, "Nonlinear dynamics of a microswimmer in poiseuille flow," Phys. Rev. Lett. 108, 218104 (2012).

[13] O A Croze, E E Ashraf, and M A Bees, "Sheared bioconvection in a horizontal tube," Physical Biology 7, 046001 (2010).

[14] William M. Durham, Eric Climent, and Roman Stocker, "Gyrotaxis in a steady vortical flow," Phys. Rev. Lett. 106, 238102 (2011).

[15] José-Agustín Arguedas-Leiva and Michael Wilczek, "Microswimmers in an axisymmetric vortex flow," New Journal of Physics 22, 053051 (2020).

[16] D. Matsunaga, F. Meng, A. Zöttl, R. Golestanian, and J. M. Yeomans, "Focusing and sorting of ellipsoidal magnetic particles in microchannels," Phys. Rev. Lett. 119, 198002 (2017).

[17] F. De Lillo, M. Cencini, W. M. Durham, M.l Barry, R. Stocker, E. Climent, and G. Boffetta, "Turbulent fluid acceleration generates clusters of gyrotactic microorganisms," Phys. Rev. Lett. 112, 044502 (2014).

[18] F. Santamaria, F. De Lillo, M. Cencini, and G. Boffetta, "Gyrotactic trapping in laminar and turbulent kolmogorov flow," Physics of Fluids 26, 111901 (2014).

[19] R. N. Bearon, "Helical swimming can provide robust upwards transport for gravitactic single-cell algae; a mechanistic model," J. Math. Biol. 66, 1341 (2013).

[20] G. J. Thorn and R. N. Bearon, “Transport of spherical gyrotactic organisms in general three-dimensional flow fields," Physics of Fluids 22, 041902 (2010).

[21] A. Aarab, M. Guedda, N. Alaa, and C. Misbah, "Mapping vesicle dynamics onto that of a rigid sphere in five dimensions," Phys. Rev. E 98, 042407 (2018).

[22] M. Guedda, M. Abaidi, M. Benlahsen, and C. Misbah, "Dynamic modes of quasispherical vesicles: Exact analytical solutions," Phys. Rev. E 86, 051915 (2012).

[23] T. J. Pedley and J. O. Kessler, "Hydrodynamic phenomena in suspensions of swimming microorganisms," Annual Review of Fluid Mechanics 24, 313-358 (1992).

[24] T. J. Pedley and J. O. Kessler, "A new continuum model for suspensions of gyrotactic micro-organisms," Journal of Fluid Mechanics 212, 155-182 (1990).

[25] F. P. Bretherton, "The motion of rigid particles in a shear flow at low reynolds number," Journal of Fluid Mechanics 14, 284-304 (1962).

[26] G. B. Jeffery, "The motion of ellipsoidal particles immersed in a viscous fluid," Proceedings of the Royal Society of London Series A 102, 161-179 (1922).

[27] E. J. Hinch and L. G. Leal, "Note on the rheology of a dilute suspension of dipolar spheres with weak brownian couples," Journal of Fluid Mechanics 56, 803-813 (1972).

[28] Borge ten Hagen, Raphael Wittkowski, and Hartmut Löwen, "Brownian dynamics of a self-propelled particle in shear flow," Phys. Rev. E 84, 031105 (2011).

[29] M. Cencini, M. Franchino, F. Santamaria, and G. Boffetta, "Centripetal focusing of gyrotactic phytoplankton," Journal of Theoretical Biology 399, 62-70 (2016).

[30] M. Cencini, G. Boffetta, M. Borgnino, and F. De Lillo, "Gyrotactic phytoplankton in laminar and turbulent flows: A dynamical systems approach," The European Physical Journal E 42, 31 (2019).

[31] R. Finken, S. Kessler, and U. Seifert, "Micro-capsules in shear flow," Journal of Physics: Condensed Matter 23, 184113 (2011).

[32] F. De Lillo, M Cencini, G Boffetta, and F Santamaria, "Geotropic tracers in turbulent flows: a proxy for fluid acceleration," Journal of Turbulence 14, 24-33 (2013). 